\title{
Bayesian estimation of the mixture of exponentiated inverted Weibull distribution using noninformative and informative priors
}

\author{
Muhammad Aslam ${ }^{1}$, Sonia Nawaz ${ }^{1}$, Sajid $\mathrm{Ali}^{2}$ and S.A. Kushani Priyangika De Silva ${ }^{3 *}$ \\ ${ }^{\prime}$ Department of Mathematics and Statistics, Riphah International University, Islamabad, Pakistan. \\ ${ }^{2}$ Department of Statistics, Quaid-i-Azam University, Islamabad, Pakistan. \\ ${ }^{3}$ Department of Mathematics, Faculty of Science, University of Peradeniya, Peradeniya.
}

Revised: 15 April 2018; Accepted: 25 May 2018

\begin{abstract}
In this study, Bayesian analysis of exponentiated inverted Weibull distribution (EIWD) is discussed. In particular, estimation of the scale parameter of the EIWD is focused whereas the shape parameter is assumed fixed. To derive the posterior distribution, uniform, Jeffreys, gamma and inverse Levy priors are used. Furthermore, to obtain Bayes estimates, the square error loss function (SELF), quadratic loss function (QLF), weighted loss function (WLF), precautionary loss function (PLF) and weighted balance loss function (WBLF) are considered. For comparison of the performance of different loss functions, the posterior risk is also calculated in this article. From application to the failure times of windshields dataset, results suggest that the uniform prior is a better prior than the Jeffreys prior, and WBLF is a suitable loss function for the estimation of the scale parameter of the mixture of EIWD. By comparing noninformative and informative priors, it is observed that the gamma prior has the minimum posterior risk.
\end{abstract}

Keywords: Bayes estimator, informative prior, loss function, mixture distribution, noninformative prior, posterior distribution.

\section{INTRODUCTION}

There is a continuous effort in statistics to introduce flexible classes of probability distributions. Corderio et al. (2013) presented the idea of adding two-parameters to a continuous distribution. This idea of adding parameters was first introduced by Lehmann (1953) and later discussed by Nadarajah and Kotz (2006), which leads to a new class of exponentiated generalised distribution that can be interpreted as a double structure of Lehmann alternatives. Several mathematical properties of this class have been obtained in literature, including the ordinary moments, generating function, mean deviation and order statistics. Moreover, the method of maximum likelihood estimation has also been investigated.

Datta (2013) compared the Weibull and exponentiated Weibull distributions and concluded that exponentiated Weibull distribution is more suitable than the Weibull distribution for wind speed data modelling. Elbatal and Muhammad (2014) derived the moment generating function and the $\mathrm{r}^{\text {th }}$ moment of the exponentiated inverted Weibull distribution (EIWD). Flaih et al. (2012) discussed some properties of the exponentiated distribution to failure times following bathtub pattern. Similarly, Gupta and Kundu (2001) studied a number of properties of the exponentiated exponential distribution. Moreover, Hassan et al. (2014) presented estimation of population parameters for the EIWD depending on equal and unequal group data. Lemonte and Corderio (2011) introduced the exponentiated generalised inverse Gaussian distribution and studied its properties.

To add flexibility to the existing probability distributions, finite mixture of lifetime models has received considerable attention of researchers. Many authors used mixture distributions in different real-life problems by using the frequentist and Bayesian estimation (Sultan et al., 2007; Al-Hussaini \& Hussein, 2012; Ali, 2014). AL-Hussaini and Hussein (2012) introduced a two-component finite mixture of the exponentiated

\footnotetext{
*Corresponding author (desilvkp@clarkson.edu; (D https://orcid.org/0000-0002-1958-3889)
} 
model and studied its properties. Ali (2014) introduced and studied the mixture of inverse Rayleigh distribution and estimated its parameters by assuming informative and noninformative priors under different loss functions.

The Weibull distribution was introduced by a Swedish scientist Wallodi Weibull in 1951 and has many applications in different fields such as medicine, ecology etc. (Rinne, 2008). This distribution and its various forms have also been considered in mixture modelling. For example, Ahmed et al. (2015) obtained Bayes estimators of the shape parameter of the exponentiated inverse Weibull distribution (EIWD) using a Bayesian technique under square error loss function, entropy loss function and the precautionary loss function. Aljuaid (2013) considered the complete and type-II censored data and obtained parameter estimates for EIWD. Li and Sedransk (1988) discussed a topological approach for mixture distributions. Sultan et al. (2007) introduced the mixture of inverse Weibull distribution and studied various properties, especially the identifiability property of the mixture model. Tahir et al. (2016) also discussed the mixture of exponential probability distributions.

The aim of this article is to introduce a twocomponent mixture of EIWD and estimate its parameters by the Bayesian approach. In particular, two different noninformative priors and five loss functions are considered to obtain the Bayes estimators. Moreover, the posterior risks under different loss functions to assess the performance of different Bayes estimators are also derived.

\section{METHODOLOGY}

\section{Two-component mixture model of the EIWD}

The cumulative distribution function (cdf) of the EIWD for a random variable $\mathrm{X}$ is defined as:

$$
F(x ; \theta, \beta)=\left(e^{-x^{-\beta}}\right)^{\theta}, \quad x>, \theta, \beta>0,
$$

where $\theta$ and $\beta$ are the shape parameters. The probability density function (pdf) of the EIWD is given as:

$$
f(x)=\theta \beta x^{-(\beta+1)}\left\{\exp \left(-x^{-\beta}\right)\right\}^{\theta} \quad x, \theta, \beta>0,
$$

To introduce a two-component mixture model of EWID, it is supposed that the population consists of two types of subpopulations and $w$ is the mixing proportion of the first type sub-population and $(1-w)$ is the second type sub-population. The resulting mixture model is given as: $f(x)=w f_{1}(x)+(1-w) f_{2}(x), \quad 0<w<1$.

Let both the sub-populations follow the EIWD with a common shape parameter $\beta$, i.e.,

$f_{k}(x)=\theta_{k} \beta x^{-(\beta+1)}\left\{\exp \left(-x^{-\beta}\right)\right\}^{\theta_{k}}, x, \theta_{k}, \beta>0, \quad k=1,2$

Then, the pdf of the mixture model is given as:

$f(x)=w \theta_{1} \beta x^{-(\beta+1)}\left\{\exp \left(-x^{-\beta}\right)\right\}^{\theta_{1}}+(1-w) \theta_{2} \beta x^{-(\beta+1)}\left\{\exp \left(-x^{-\beta}\right)\right\}^{\theta_{2}}$,

Similarly, the cdf for the two-component mixture model is given by:

$F(x)=w F_{1}(x)+(1-w) F_{2}(x) \quad 0<w<1$

where

$F_{k}(x)=\left(e^{-x^{-\beta}}\right)^{\theta_{k}}, \quad x, \theta_{k}, \beta>0 \quad k=1,2$

Thus,

$$
\begin{gathered}
F(x)=w\left\{\exp \left(-x^{-\beta}\right)\right\}^{\theta_{1}}+(1-w)\left\{\exp \left(-x^{-\beta}\right)\right\}^{\theta_{2}} ; \\
x, \theta_{1}, \theta_{2}, \beta>0, \quad 0<w<1
\end{gathered}
$$

The graphical depiction of the mixture of EIWD for different parameter combinations is given in Figure 1.

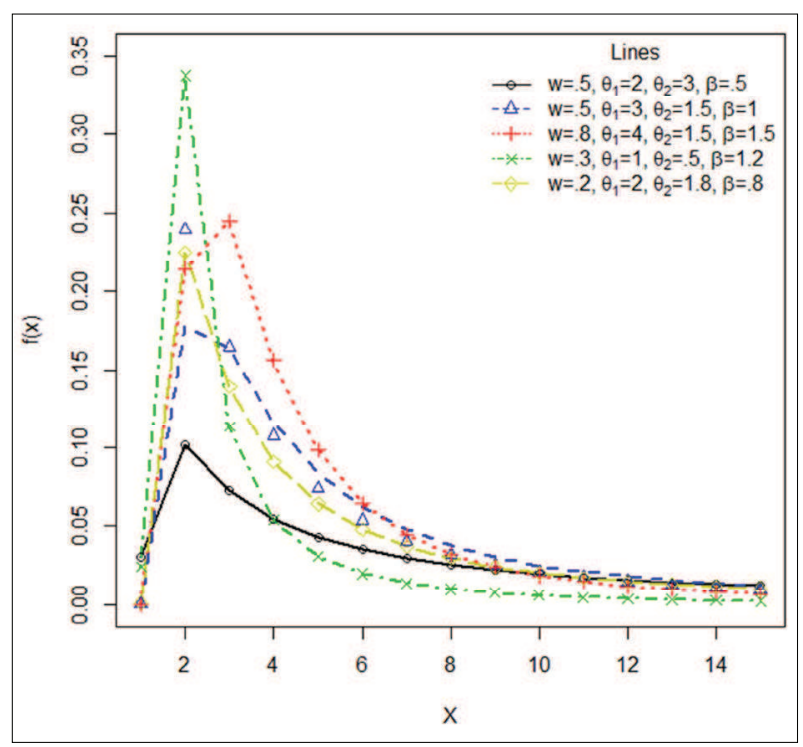

Figure 1: Probability density function of the mixture of EIWD 
Suppose that $n$ elements are placed on a life testing experiment. Let $\mathrm{r}$ out of $\mathrm{n}$ be the elements having a lifetime $\left[0, t_{0}\right]$, where $t_{0}$ is the pre-set termination time and the rest of the $(n-r)$ elements are still functioning at the termination of the experiment. Further, let each subpopulation have $r_{k}$ failed units and the rest as $n=n_{1}+n_{2}$, $r=r_{1}+r_{2}$ and $k=1,2$. Let $x_{k j}$ denote the failure time of the $j^{\text {th }}$ element belonging to the $k^{\text {th }}$ subpopulation, where $j=1,2, \ldots, r_{k}, 0<x_{k j} \leq t_{0}$ and $k=1,2$. For such conditions, the likelihood function (Ali, 2014) is defined as:

$L\left(\theta_{1}, \theta_{2}, \beta, w \mid \mathbf{x}\right) \propto\left\{\prod_{j=1}^{r_{1}} f w_{1}\left(x_{1 j}\right)\right\}\left\{\prod_{j=1}^{r_{2}}(1-w) f_{2}\left(x_{2 j}\right)\right\}\left\{1-F\left(t_{0}\right)\right\}^{n-r}$,

For the mixture of EWID, the likelihood function gets the form:

$$
\begin{aligned}
& L\left(\theta_{1}, \theta_{2}, w \mid \mathbf{x}\right) \propto\{\left.\prod_{j=1}^{r_{1}} w \theta_{1} \beta x_{1 j}{ }^{-(\beta+1)}\left\{\exp \left(-x_{1 j}{ }^{-\beta}\right)\right\}^{\theta_{1}}\right\}\left\{\prod_{j=1}^{r_{2}}(1-w) \theta_{2} \beta x_{2 j}{ }^{-(\beta+1)}\left\{\exp \left(-x_{2 j}{ }^{-\beta}\right)\right\}^{\theta_{2}}\right\} \\
& \times {\left[1-\left\{w\left\{\exp \left(-t_{0}^{-\beta}\right)\right\}^{\theta_{1}}+(1-w)\left\{\exp \left(-t_{0}^{-\beta}\right)\right\}^{\theta_{2}}\right\}\right]^{n-r} } \\
& L\left(\theta_{1}, \theta_{2}, w \mid \mathbf{x}\right) \propto \sum_{k=0}^{n-r} \sum_{l=0}^{k}(-1)^{k}\left(\begin{array}{c}
n-r \\
k
\end{array}\right)\left(\begin{array}{c}
k \\
l
\end{array}\right) \theta_{1}^{r_{1}} \theta_{2}^{r_{2}} w^{r_{1}+k-l}(1-w)^{r_{2}+l} \\
& \times \exp \left[-\theta_{1}\left\{\sum_{j=1}^{r_{1}} w_{1 j}+v(k-l)\right\}\right] \exp \left[-\theta_{2}\left\{\sum_{j=1}^{r_{2}} w_{2 j}+v l\right\}\right]
\end{aligned}
$$

where $w_{1 j}=x_{1 j}^{-\beta}, w_{2 j}=x_{2 j}^{-\beta}, v=t_{0}^{-\beta}$

\section{Posterior distribution using informative gamma prior}

In addition to the current/observed information, Bayesian methodology utilises the prior information and different types of priors are available in literature. Informative priors represent definite knowledge about a problem under study. In this section, gamma prior is considered as an informative prior. The prior distributions can be expressed as: $p\left(\theta_{1}\right)=\frac{b_{1}^{a_{1}}}{\Gamma\left(a_{1}\right)} \theta^{a_{1}-1} e^{-b_{1}} \theta_{1} ; p\left(\theta_{2}\right)=\frac{b_{2}^{a_{2}}}{\Gamma\left(a_{2}\right)} \theta^{a_{2}-1} e^{-b_{2}} \theta_{2}$; and $w \sim U(0,1)$. The joint prior distribution of $\theta_{1}, \theta_{2}$ and $w$ is given in equation (8):

$$
p\left(\theta_{1}, \theta_{2}, w\right) \propto \theta_{1}^{a_{1}-1} \theta_{2}^{a_{2}-1} e^{-b_{1} \theta_{1}-b_{2} \theta_{2}} ; \theta_{1}, \theta_{2}>0,0<w<1
$$

where $a_{1}, a_{2}, b_{1}$ and $b_{2}$ are the parameters of the prior distribution and known as the hyperparameters. The joint posterior distribution of $\theta_{1}, \theta_{2}$ and $w$ is calculated by the celebrated Bayes theorem as:

$$
\begin{aligned}
& p\left(\theta_{1}, \theta_{2}, w \mid \mathbf{x}\right)=\frac{p\left(\theta_{1}, \theta_{2}, w\right) L\left(\theta_{1}, \theta_{2}, w \mid x\right)}{\iint_{0}^{\infty} \int_{0}^{\infty} p\left(\theta_{1}, \theta_{2}, w\right) L\left(\theta_{1}, \theta_{2}, w \mid x\right) d \theta_{1} d \theta_{2} d w} \\
& p\left(\theta_{1}, \theta_{2}, w \mid x\right) \propto \sum_{k=0}^{n-r} \sum_{l=0}^{k}(-1)^{k}\left(\begin{array}{c}
n-r \\
k
\end{array}\right)\left(\begin{array}{c}
k \\
l
\end{array}\right) \theta_{1}^{r_{1}+a_{1}-1} \theta_{2}^{r_{2}+a_{2}-1} w^{r_{1}+k-l}(1-w)^{r_{2}+l}
\end{aligned}
$$




$$
\begin{aligned}
& \times \exp \left[-\theta_{1}\left\{b_{1}+\sum_{j=1}^{r_{1}} w_{1 j}+v(k-l)\right\}\right] \\
& \times \exp \left[-\theta_{2}\left\{b_{2}+\sum_{j=1}^{r_{2}} w_{2 j}+v(l)\right\} ; \quad \theta_{1}, \theta_{2}>0,\right. \\
& p\left(\theta_{1}, \theta_{2}, w \mid \mathbf{x}\right)=\frac{1}{C_{3}} \sum_{k=0}^{n-r} \sum_{l=0}^{k}(-1)^{k}\left(\begin{array}{c}
n-r \\
k
\end{array}\right)\left(\begin{array}{l}
k \\
l
\end{array}\right) \\
& \theta_{1}^{r_{1}+a_{1}-1} \theta_{2}^{r_{2}+a_{2}-1} w^{r_{1}+k-l}(1-w)^{r_{2}+l} \\
& \quad \times \exp \left[-\theta_{1}\left\{b_{1}+\sum_{j=1}^{r_{1}} w_{1 j}+(k-l) v\right\}-\right. \\
& \left.\theta_{2}\left\{b_{2}+\sum_{j=1}^{r_{2}} w_{2 j}+(l) v\right\}\right] \theta_{1}, \theta_{2}>0,0<w<1,
\end{aligned}
$$

where

$$
\begin{aligned}
C_{3}= & \int_{0}^{\infty} \int_{0}^{\infty} \int_{0}^{1} \sum_{k=0}^{n-r} \sum_{l=0}^{k}(-1)^{k}\left(\begin{array}{c}
n-r \\
k
\end{array}\right)\left(\begin{array}{l}
k \\
l
\end{array}\right) \\
& \theta_{1}^{r_{1}+a_{1}-1} \theta_{2}^{r_{2}+a_{2}-1} w^{r_{1}+k-l}(1-w)^{r_{2}+l} \\
& \times \exp \left[-\theta_{1}\left\{b_{1}+\sum_{j=1}^{r_{1}} w_{1 j}+v(k-l)\right\}-\right. \\
C_{3}= & \sum_{k=0}^{n-r} \sum_{l=0}^{k}(-1)^{k}\left(\begin{array}{c}
n-r \\
k
\end{array}\right)\left(\begin{array}{l}
k \\
l
\end{array}\right) \\
\beta\left(r_{1}+k-l+1, r_{2}+l+1\right) \times & \Gamma\left(r_{1}+a_{1}\right) \Gamma\left(r_{2}+a_{2}\right) \\
\left.\frac{r_{2}}{\left\{b_{1}\right.}+\sum_{j=1}^{r_{1}} w_{1 j}+v(k-l)\right\} d w d \theta_{2} d \theta_{1} & \left\{b_{2}+\sum_{j=1}^{r_{2}} w_{2 j}+v(l)\right\}
\end{aligned}
$$

and $\beta(a, b)=\frac{\Gamma(a) \Gamma(b)}{\Gamma(a+b)}$ is the beta function. In the next section, marginal posterior distributions of each parameter are derived.

\section{The marginal posterior distributions using GP}

In this section, the derivation of the marginal posterior distribution of each parameter by integrating out the nuisance parameter is discussed. The marginal posterior distribution of $\theta_{1}$ using GP is obtained as:

$$
\begin{aligned}
& p\left(\theta_{1} \mid x\right)=\frac{1}{C_{3}} \sum_{k=0}^{n-r} \sum_{l=0}^{k}(-1)^{k}\left(\begin{array}{c}
n-r \\
k
\end{array}\right)\left(\begin{array}{l}
k \\
l
\end{array}\right) \\
& \beta\left(r_{1}+k-l+1, r_{2}+l+1\right) \\
& \times \frac{\Gamma\left(r_{2}+a_{2}\right)}{\left\{b_{2}+\sum_{j=1}^{r_{1}} w_{2 j}+v(l)\right\}^{\left(r_{2}+a_{2}\right)}} \theta_{1}^{r_{1}+a_{1}-1} \\
& \exp \left\lfloor-\theta_{1}\left\{b_{1}+\sum_{j=1}^{r_{2}} w_{1 j}+v(k-l)\right\} ; ;\right.
\end{aligned}
$$

The marginal posterior distribution of $\theta_{2}$ is given by:

$$
\begin{aligned}
& p\left(\theta_{2} \mid x\right)=\frac{1}{C_{3}} \sum_{k=0}^{n-r} \sum_{l=0}^{k}(-1)^{k}\left(\begin{array}{c}
n-r \\
k
\end{array}\right)\left(\begin{array}{l}
k \\
l
\end{array}\right) \\
& \beta\left(r_{1}+k-l+1, r_{2}+l+1\right) \\
& \times \frac{\Gamma\left(r_{1}+a_{1}\right)}{\left\{b_{1}+\sum_{j=1}^{r_{1}} w_{1 j}+v(k-l)\right\}^{\left(r_{1}+a_{1}\right)}} \theta_{2}^{r_{2}+a_{2}-1} \\
& \exp \left[-\theta_{2}\left\{b_{2}+\sum_{j=1}^{r_{2}} w_{2 j}+v(l)\right\}\right] ;
\end{aligned}
$$

The marginal posterior distribution of $w$ is given by:

$$
\begin{aligned}
& p(w \mid x)=\frac{1}{C_{3}} \sum_{k=0}^{n-r} \sum_{l=0}^{k}(-1)^{k}\left(\begin{array}{c}
n-r \\
k
\end{array}\right)\left(\begin{array}{l}
k \\
l
\end{array}\right) \\
& \times \frac{\Gamma\left(r_{1}+a_{1}\right) \Gamma\left(r_{2}+a_{2}\right) w^{r_{1}+k-l}(1-w)^{r_{2}+l}}{\left\{b_{1}+\sum_{j=1}^{r_{1}} w_{1 j}+v(k-l)\right\}^{\left(r_{1}+a_{1}\right)}\left\{b_{2}+\sum_{j=1}^{r_{2}} w_{2 j}+v(l)\right\}^{\left(r_{2}+a_{2}\right)}} ;
\end{aligned}
$$

$0<w<1$

In the next section, the derivation of the Bayes estimators is discussed. 


\section{Bayesian estimation}

The aim of this section is to derive Bayes estimators of the mixture of EWID and for this, five different loss functions, namely, square error loss function (SELF), quadratic loss function (QLF), weighted loss function (WLF), precautionary loss function (PLF) and weighted balance loss function (WBLF) are considered. To this end, the derivation of Bayes estimators and their posterior risks assuming the GP under the above-mentioned loss functions is presented. The Bayes estimator $d^{*}$ is derived by minimising the posterior expected loss, defined as $\mathfrak{R}\left(d^{*}\right)=E_{\theta \mid \mathbf{x}}\left\{L\left(\theta, d^{*}\right)\right\}$, where $L\left(\theta, d^{*}\right)$ is the loss incurred estimating $\theta$ by $d^{*}$. For a given prior, the general form of the Bayes estimators (BEs) and posterior risks (PRs) under SELF, QLF, WLF, PLF and WBLF are given in Table 1 (Ali et al., 2014).

Table 1: Bayes estimators and posterior risks under SELF, PLF and DLF

\begin{tabular}{lll}
\hline Loss function & Bayes estimators (BEs) & Posterior risks (PRs) \\
\hline$S E L F=L\left(\theta, d^{*}\right)=\left(\theta-d^{*}\right)^{2}$ & $d^{*}=E_{\theta \mid \mathbf{x}}(\theta)$ & $\mathfrak{R}\left(d^{*}\right)=E_{\theta \mid \mathbf{x}}\left(\theta^{2}\right)-\left\{E_{\theta \mid \mathbf{x}}(\theta)\right\}^{2}$ \\
$Q L F=L\left(\theta, d^{*}\right)=\left(\frac{\theta-d^{*}}{\theta}\right)^{2}$ & $d^{*}=\left\{E_{\theta \mid \mathbf{x}}\left(\theta^{-2}\right)\right\}^{-1} E_{\theta \mid \mathbf{x}}\left(\theta^{-1}\right)$ & $\mathfrak{R}\left(d^{*}\right)=1-\left\{E_{\theta \mid \mathbf{x}}\left(\theta^{-2}\right)\right\}^{-1}\left\{E_{\theta \mid \mathbf{x}}\left(\theta^{-1}\right)\right\}^{2}$ \\
$W L F=L\left(\theta, d^{*}\right)=\frac{\left(\theta-d^{*}\right)^{2}}{\theta}$ & $d^{*}=\left\{E_{\theta \mid \mathbf{x}}\left(\theta^{-1}\right)\right\}^{-1}$ & $\mathfrak{R}\left(d^{*}\right)=E_{\theta \mid \mathbf{x}}(\theta)-\left\{E_{\theta \mid \mathbf{x}}\left(\theta^{-1}\right)\right\}^{-1}$ \\
$P L F=L\left(\theta, d^{*}\right)=\frac{\left(\theta-d^{*}\right)^{2}}{d^{*}}$ & $d^{*}=\left\{E_{\theta \mid \mathbf{x}}\left(\theta^{2}\right)\right\}^{\frac{1}{2}}$ & $\mathfrak{R}\left(d^{*}\right)=2\left\{E_{\theta \mid \mathbf{x}}\left(\theta^{2}\right)\right\}^{\frac{1}{2}}-2 E_{\theta \mid \mathbf{x}}(\theta)$ \\
$W B L F=L\left(\theta, d^{*}\right)=\left(\frac{\theta-d^{*}}{d^{*}}\right)^{2}$ & $d^{*}=\left\{E_{\theta \mid \mathbf{x}}(\theta)\right\}^{-1} E_{\theta \mid \mathbf{x}}\left(\theta^{2}\right)$ & $\Re\left(d^{*}\right)=1-\left\{E_{\theta \mid \mathbf{x}}(\theta)\right\}^{2}\left\{E_{\theta \mid \mathbf{x}}\left(\theta^{2}\right)\right\}^{-1}$
\end{tabular}

Taking $d^{*}=\theta_{1}^{*}, \theta_{2}^{*}$ and $w^{*}$ for estimating the parameters $\theta=\theta_{1}, \theta_{2}$ and $w_{1}$, the expressions for the Bayes estimators and posterior risks are given in the following subsections:

\section{Bayes estimators and the Bayes risks under SELF}

The Bayes estimator of the parameter $\theta_{1}$ under SELF using equation (10) is obtained as:

$$
\begin{aligned}
& \theta_{1}^{*}=\frac{1}{C_{3}} \sum_{k=0}^{n-r} \sum_{l=0}^{k}(-1)^{k}\left(\begin{array}{c}
n-r \\
k
\end{array}\right)\left(\begin{array}{l}
k \\
l
\end{array}\right) \\
& \frac{\beta\left(r_{1}+k-l+1, r_{2}+l+1\right) \Gamma\left(r_{1}+a_{1}+1\right) \Gamma\left(r_{2}+a_{2}\right)}{\left\{b_{1}+\sum_{j=1}^{r_{1}} w_{1 j}+v(k-l)\right\}^{r_{1}+a_{1}+1}\left\{b_{2}+\sum_{j=1}^{r_{2}} w_{2 j}+v(l)\right\}^{r_{2}}}
\end{aligned}
$$

The Bayes risk of $\theta_{1}$ under SELF is obtained as:
$\mathfrak{R}\left(\theta_{1}^{*}\right)=\frac{1}{C_{3}} \sum_{k=0}^{n-r} \sum_{l=0}^{k}(-1)^{k}\left(\begin{array}{c}n-r \\ k\end{array}\right)\left(\begin{array}{l}k \\ l\end{array}\right)$

$\frac{\beta\left(r_{1}+k-l+1, r_{2}+l+1\right) \Gamma\left(r_{1}+a_{1}+2\right) \Gamma\left(r_{2}+a_{2}\right)}{\left\{b_{1}+\sum_{j=1}^{r_{1}} w_{1 j}+v(k-l)\right\}^{\left(r_{1}+a_{1}+2\right)}\left\{b_{2}+\sum_{j=1}^{r_{2}} w_{2 j}+v(l)\right\}^{\left(r_{2}+a_{2}\right)}}$

$-\left[\frac{1}{C_{3}} \sum_{k=0}^{n-r} \sum_{l=0}^{k}(-1)^{k}\left(\begin{array}{c}n-r \\ k\end{array}\right)\left(\begin{array}{l}k \\ l\end{array}\right)\right.$

$\left.\frac{\beta\left(r_{1}+k-l+1, r_{2}+l+1\right) \Gamma\left(r_{1}+a_{1}+1\right) \Gamma\left(r_{2}+a_{2}\right)}{\left\{b_{1}+\sum_{j=1}^{r_{1}} w_{1 j}{ }^{-1}+v(k-l)\right\}^{\left(r_{1}+a_{1}+1\right)}\left\{b_{2}+\sum_{j=1}^{r_{2}} w_{2 j}+v(l)\right\}^{\left(r_{2}+a_{2}\right)}}\right]$ 
The Bayes estimator of the parameter $\theta_{2}$ under SELF using equation (11) is obtained as:

$$
\begin{aligned}
& \theta_{2}^{*}=\frac{1}{C_{3}} \sum_{k=0}^{n-r} \sum_{l=0}^{k}(-1)^{k}\left(\begin{array}{c}
n-r \\
k
\end{array}\right)\left(\begin{array}{l}
k \\
l
\end{array}\right) \\
& \frac{\beta\left(r_{1}+k-l+1, r_{2}+l+1\right) \Gamma\left(r_{1}+a_{1}\right) \Gamma\left(r_{2}+a_{2}+1\right)}{\left\{b_{1}+\sum_{j=1}^{r_{1}} w_{1 j}+v(k-l)\right\}^{\left(r_{1}+a_{1}\right)}\left\{b_{2}+\sum_{j=1}^{r_{2}} w_{2 j}+v(l)\right\}^{\left(r_{2}+a_{2}+1\right)}}
\end{aligned}
$$

The Bayes risk of $\theta_{2}$ under SELF is obtained as:

$$
\begin{aligned}
& \Re\left(\theta_{2}^{*}\right)=\frac{1}{C_{3}} \sum_{k=0}^{n-r} \sum_{l=0}^{k}(-1)^{k}\left(\begin{array}{c}
n-r \\
k
\end{array}\right)\left(\begin{array}{l}
k \\
l
\end{array}\right) \\
& \frac{\beta\left(r_{1}+k-l+1, r_{2}+l+1\right) \Gamma\left(r_{1}+a_{1}\right) \Gamma\left(r_{2}+a_{2}+3\right)}{\left\{b_{1}+\sum_{j=1}^{r_{1}} w_{1 j}+v(k-l)\right\}^{\left(r_{1}+a_{1}\right)}\left\{b_{2}+\sum_{j=1}^{r_{2}} w_{2 j}+v(l)\right\}^{\left(r_{2}+a_{2}+3\right)}} \\
& -\left[\frac{1}{C_{3}} \sum_{k=0}^{n-r} \sum_{l=0}^{k}(-1)^{k}\left(\begin{array}{c}
n-r \\
k
\end{array}\right)\left(\begin{array}{l}
k \\
l
\end{array}\right) .\right. \\
& \left.\frac{\beta\left(r_{1}+k-l+1, r_{2}+l+1\right) \Gamma\left(r_{1}+a_{1}\right) \Gamma\left(r_{2}+a_{2}+1\right)}{\left\{b_{1}+\sum_{j=1}^{r_{1}} w_{1 j}+v(k-l)\right\}^{\left(r_{1}+a_{1}\right)}\left\{b_{2}+\sum_{j=1}^{r_{2}} w_{2 j}+v(l)\right\}^{\left(r_{2}+a_{2}+1\right)}}\right]^{2}
\end{aligned}
$$

The Bayes estimator of the parameter $w$ under SELF using equation (12) is obtained as:

$$
\begin{aligned}
& w^{*}=\frac{1}{C_{3}} \sum_{k=0}^{n-r} \sum_{l=0}^{k}(-1)^{k}\left(\begin{array}{c}
n-r \\
k
\end{array}\right)\left(\begin{array}{l}
k \\
l
\end{array}\right) \\
& \frac{\beta\left(r_{1}+k+2, r_{2}+l+1\right) \Gamma\left(r_{1}+a_{1}\right) \Gamma\left(r_{2}+a_{2}\right)}{\left\{b_{1}+\sum_{j=1}^{r_{1}} w_{1 j}+v(k-l)\right\}^{\left(r_{1}+a_{1}\right)}\left\{b_{2}+\sum_{j=1}^{r_{2}} w_{2 j}+v(l)\right\}^{\left(r_{2}+a_{2}\right)}}
\end{aligned}
$$

The Bayes risk of $w$ under SELF is obtained as:

$$
\mathfrak{R}\left(w^{*}\right)=\frac{1}{C_{3}} \sum_{k=0}^{n-r} \sum_{l=0}^{k}(-1)^{k}\left(\begin{array}{c}
n-r \\
k
\end{array}\right)\left(\begin{array}{c}
k \\
l
\end{array}\right)
$$

$$
\frac{\beta\left(r_{1}+k+3, r_{2}+l+1\right) \Gamma\left(r_{1}+a_{1}\right) \Gamma\left(r_{2}+a_{2}\right)}{\left\{b_{1}+\sum_{j=1}^{r_{1}} w_{1 j}+v(k-l)\right\}^{\left(r_{1}+a_{1}\right)}\left\{b_{2}+\sum_{j=1}^{r_{2}} w_{2 j}+v(l)\right\}^{\left(r_{2}+a_{2}\right)}}
$$

$-\left[\frac{1}{C_{3}} \sum_{k=0}^{n-r} \sum_{l=0}^{k}(-1)^{k}\left(\begin{array}{c}n-r \\ k\end{array}\right)\left(\begin{array}{c}k \\ l\end{array}\right)\right.$

$$
\left.\frac{\beta\left(r_{1}+k+2, r_{2}+l+1\right) \Gamma\left(r_{1}+a_{1}\right) \Gamma\left(r_{2}+a_{2}\right)}{\left\{b_{1}+\sum_{j=1}^{r_{1}} w_{1 j}+v(k-l)\right\}^{\left(r_{1}+a_{1}\right)}\left\{b_{2}+\sum_{j=1}^{r_{2}} w_{2 j}+v(l)\right\}^{\left(r_{2}+a_{2}\right)}}\right]^{2}
$$

\section{Bayes estimators and the Bayes risks under QLF}

The Bayes estimator of the parameter $\theta_{1}$ under QLF using equation (10) is obtained as :

$$
\theta_{1}^{*}=\frac{\sum_{k=0}^{n-r} \sum_{l=0}^{k}(-1)^{k}\left(\begin{array}{c}
n-r \\
k
\end{array}\right)\left(\begin{array}{c}
k \\
l
\end{array}\right) \frac{\beta\left(r_{1}+k-l+1, r_{2}+l+1\right) \Gamma\left(r_{1}+a_{1}-1\right) \Gamma\left(r_{2}+a_{2}\right)}{\left\{b_{1}+\sum_{j=1}^{r_{1}} w_{1 j}+v(k-l)\right\}^{\left(r_{1}+a_{1}-1\right)}\left\{b_{2}+\sum_{j=1}^{r_{2}} w_{2 j}+v(l)\right\}^{\left(r_{2}+a_{2}\right)}}}{\sum_{k=0}^{n-r} \sum_{l=0}^{k}(-1)^{k}\left(\begin{array}{c}
n-r \\
k
\end{array}\right)\left(\begin{array}{c}
k \\
l
\end{array}\right) \frac{\beta\left(r_{1}+k-l+1, r_{2}+l+1\right) \Gamma\left(r_{1}+a_{1}-2\right) \Gamma\left(r_{2}+a_{2}\right)}{\left\{b_{1}+\sum_{j=1}^{r_{1}} w_{1 j}+v(k-l)\right\}^{\left(r_{1}+a-2\right)}\left\{b_{2}+\sum_{j=1}^{r_{2}} w_{2 j}+v(l)\right\}^{\left(r_{2}+a_{2}\right)}}}
$$


The Bayes risk of $\theta_{1}$ under QLF is obtained as:

$$
\mathfrak{R}\left(\theta_{1}^{*}\right)=1-\frac{\frac{1}{C_{3}}\left[\sum_{k=0}^{n-r} \sum_{l=0}^{k}(-1)^{k}\left(\begin{array}{c}
n-r \\
k
\end{array}\right)\left(\begin{array}{l}
k \\
l
\end{array}\right) \frac{\beta\left(r_{1}+k-l+1, r_{2}+l+1\right) \Gamma\left(r_{1}+a_{1}-1\right) \Gamma\left(r_{2}+a_{2}\right)}{\left\{b_{1}+\sum_{j=1}^{r_{1}} w_{1 j}+v(k-l)\right\}^{\left(r_{1}+a_{1}-1\right)}\left\{b_{2}+\sum_{j=1}^{r_{2}} w_{2 j}+v(l)\right\}^{\left(r_{2}+a_{2}\right)}}\right]^{2}}{\sum_{k=0}^{n-r} \sum_{l=0}^{k}(-1)^{k}\left(\begin{array}{c}
n-r \\
k
\end{array}\right)\left(\begin{array}{c}
k \\
l
\end{array}\right) \frac{\beta\left(r_{1}+k-l+1, r_{2}+l+1\right) \Gamma\left(r_{1}+a_{1}-2\right) \Gamma\left(r_{2}+a_{2}\right)}{\left\{b_{1}+\sum_{j=1}^{r_{1}} w_{1 j}+v(k-l)\right\}^{\left(r_{1}+a_{1}-2\right)}\left\{b_{2}+\sum_{j=1}^{r_{2}} w_{2 j}+v(l)\right\}^{\left(r_{2}+a_{2}\right)}}}
$$

The Bayes estimator of the parameter $\theta_{2}$ under QLLF using equation (11) is obtained as:

$$
\theta_{2}^{*}=\frac{\sum_{k=0}^{n-r} \sum_{l=0}^{k}(-1)^{k}\left(\begin{array}{c}
n-r \\
k
\end{array}\right)\left(\begin{array}{l}
k \\
l
\end{array}\right) \frac{\beta\left(r_{1}+k-l+1, r_{2}+l+1\right) \Gamma\left(r_{1}+a_{1}\right) \Gamma\left(r_{2}+a_{2}-1\right)}{\left\{b_{1}+\sum_{j=1}^{r_{1}} w_{1 j}+v(k-l)\right\}^{\left(r_{1}+a_{1}\right)}\left\{b_{2}+\sum_{j=1}^{r_{2}} w_{2 j}+v(l)\right\}^{\left(r_{2}+a_{2}-1\right)}}}{\sum_{k=0}^{n-r} \sum_{l=0}^{k}(-1)^{k}\left(\begin{array}{c}
n-r \\
k
\end{array}\right)\left(\begin{array}{l}
k \\
l
\end{array}\right) \frac{\beta\left(r_{1}+k-l+1, r_{2}+l+1\right) \Gamma\left(r_{1}+a_{1}\right) \Gamma\left(r_{2}+a_{2}-2\right)}{\left\{b_{1}+\sum_{j=1}^{r_{1}} w_{1 j}+v(k-l)\right\}^{\left(r_{1}+a_{1}\right)}\left\{b_{2}+\sum_{j=1}^{r_{2}} w_{2 j}+v(l)\right\}^{\left(r_{2}+a_{2}-2\right)}}}
$$

The Bayes risk of $\theta_{2}$ under QLF is obtained as:

$$
\mathfrak{R}\left(\theta_{2}^{*}\right)=1-\frac{\frac{1}{C_{3}} \sum_{k=0}^{n-r} \sum_{l=0}^{k}(-1)^{k}\left(\begin{array}{c}
n-r \\
k
\end{array}\right)\left(\begin{array}{l}
k \\
l
\end{array}\right) \frac{\beta\left(r_{1}+k-l+1, r_{2}+l+1\right) \Gamma\left(r_{1}+a_{1}\right) \Gamma\left(r_{2}+a_{2}-1\right)}{\left\{b_{1}+\sum_{j=1}^{r_{1}} w_{1 j}+v(k-l)\right\}^{r_{1}+a_{1}}\left\{b_{2}+\sum_{j=1}^{r_{2}} w_{2 j}+v(l)\right\}^{r_{2}+a_{2}-1}}}{\sum_{k=0}^{n-r} \sum_{l=0}^{k}(-1)^{k}\left(\begin{array}{c}
n-r \\
k
\end{array}\right)\left(\begin{array}{l}
k \\
l
\end{array}\right) \frac{\beta\left(r_{1}+k-l+1, r_{2}+l+1\right) \Gamma\left(r_{1}+a_{1}\right) \Gamma\left(r_{2}+a_{2}-1\right)}{\left\{b_{1}+\sum_{j=1}^{r_{1}} w_{1 j}+v(k-l)\right\}^{r_{1}+a_{1}}\left\{b_{2}+\sum_{j=1}^{r_{2}} w_{2 j}+v(l)\right\}^{r_{2}+a_{2}-1}}}
$$

The Bayes estimator of the parameter $w$ under QLF using equation (12) is obtained as:

$$
w^{*}=\frac{\sum_{k=0}^{n-r} \sum_{l=0}^{k}(-1)^{k}\left(\begin{array}{c}
n-r \\
k
\end{array}\right)\left(\begin{array}{l}
k \\
l
\end{array}\right) \frac{\beta\left(r_{1}+k-l, r_{2}+l+1\right) \Gamma\left(r_{1}+a_{1}\right) \Gamma\left(r_{2}+a_{2}\right)}{\left\{b_{1}+\sum_{j=1}^{r_{1}} w_{1 j}+v(k-l)\right\}^{\left(r_{1}+a_{1}\right)}\left\{b_{2}+\sum_{j=1}^{r_{2}} w_{2 j}+v(l)\right\}^{\left(r_{2}+a_{2}\right)}}}{\sum_{k=0}^{n-r} \sum_{l=0}^{k}(-1)^{k}\left(\begin{array}{c}
n-r \\
k
\end{array}\right)\left(\begin{array}{c}
k \\
l
\end{array}\right) \frac{\beta\left(r_{1}+k-l-1, r_{2}+l+1\right) \Gamma\left(r_{1}+a_{1}\right) \Gamma\left(r_{2}+a_{2}\right)}{\left\{b_{1}+\sum_{j=1}^{r_{1}} w_{1 j}+v(k-l)\right\}^{\left(r_{1}+a_{1}\right)}\left\{b_{2}+\sum_{j=1}^{r_{2}} w_{2 j}+v(l)\right\}^{\left(r_{2}+a_{2}\right)}}}
$$

The Bayes risk of $w$ under QLF is obtained as: 


$$
\mathfrak{R}\left(\theta_{1}^{*}\right)=1-\frac{\frac{1}{C_{3}}\left[\sum_{k=0}^{n-r} \sum_{l=0}^{k}(-1)^{k}\left(\begin{array}{c}
n-r \\
k
\end{array}\right)\left(\begin{array}{l}
k \\
l
\end{array}\right) \frac{\beta\left(r_{1}+k-l, r_{2}+l+1\right) \Gamma\left(r_{1}+a_{1}\right) \Gamma\left(r_{2}+a_{2}\right)}{\left\{b_{1}+\sum_{j=1}^{r_{1}} w_{1 j}+v(k-l)\right\}^{\left(r_{1}+a_{1}\right)}\left\{b_{2}+\sum_{j=1}^{r_{2}} w_{2 j}+v(l)\right\}^{\left(r_{2}+a_{2}\right)}}\right]^{2}}{\sum_{k=0}^{n-r} \sum_{l=0}^{k}(-1)^{k}\left(\begin{array}{c}
n-r \\
k
\end{array}\right)\left(\begin{array}{c}
k \\
l
\end{array}\right) \frac{\beta\left(r_{1}+k-l-1, r_{2}+l+1\right) \Gamma\left(r_{1}+a_{1}\right) \Gamma\left(r_{2}+a_{2}\right)}{\left\{b_{1}+\sum_{j=1}^{r_{1}} w_{1 j}+v(k-l)\right\}^{\left(r_{1}+a_{1}\right)}\left\{b_{2}+\sum_{j=1}^{r_{2}} w_{2 j}+v(l)\right\}^{\left(r_{2}+a_{2}\right)}}}
$$

\section{Bayes estimators and the Bayes risks under precautionary loss function}

The Bayes estimator of the parameter $\theta_{1}$ under PLF using equation (10) is obtained as:

$$
\theta_{1}^{*}=\left[\frac{1}{C_{3}} \sum_{k=0}^{n-r} \sum_{l=0}^{k}(-1)^{k}\left(\begin{array}{c}
n-r \\
k
\end{array}\right)\left(\begin{array}{c}
k \\
l
\end{array}\right) \frac{\beta\left(r_{1}+k-l+1, r_{2}+l+1\right) \Gamma\left(r_{1}+a_{1}+2\right) \Gamma\left(r_{2}+a_{2}\right)}{\left\{\sum_{j=1}^{r_{1}} w_{1 j}+v(k-l)\right\}^{\left(r_{1}+a_{1}+2\right)}\left\{\sum_{j=1}^{r_{2}} w_{2 j}+v(l)\right\}^{\left(r_{2}+a_{2}\right)}}\right]^{\frac{1}{2}}
$$

The Bayes risk of $\theta_{1}$ under PLF is obtained as:

$$
\begin{aligned}
& \mathfrak{R}\left(\theta_{1}^{*}\right)=\left[\frac{4}{C_{3}} \sum_{k=0}^{n-r} \sum_{l=0}^{k}(-1)^{k}\left(\begin{array}{c}
n-r \\
k
\end{array}\right)\left(\begin{array}{c}
k \\
l
\end{array}\right) \frac{\beta\left(r_{1}+k-l+1, r_{2}+l+1\right) \Gamma\left(r_{1}+a_{1}+2\right) \Gamma\left(r_{2}+a_{2}\right)}{\left.b_{1}+\sum_{j=1}^{r_{1}} w_{1 j}+v(k-l)\right]^{\left(r_{1}+a_{1}+2\right)}\left\{b_{2}+\sum_{j=1}^{r_{2}} w_{2 j}+v(l)\right\}^{\left(r_{2}+a_{2}\right)}}\right]^{\frac{1}{2}} \\
& -\frac{2}{C_{3}} \sum_{k=0}^{n-r} \sum_{l=0}^{k}(-1)^{k}\left(\begin{array}{c}
n-r \\
k
\end{array}\right)\left(\begin{array}{c}
k \\
l
\end{array}\right) \frac{\beta\left(r_{1}+k-l+1, r_{2}+l+1\right) \Gamma\left(r_{1}+a_{1}+1\right) \Gamma\left(r_{2}+a_{2}\right)}{\left[b_{1}+\sum_{j=1}^{r_{1}} w_{1 j}+v(k-l)\right]^{\left(r_{1}+a_{1}+1\right)}\left\{b_{2}+\sum_{j=1}^{r_{2}} w_{2 j}+v(l)\right\}^{\left(r_{2}+a_{2}\right)}}
\end{aligned}
$$

The Bayes estimator of the parameter $\theta_{2}$ under PLF using equation (11) is obtained as:

$$
\theta_{2}{ }^{*}=\left[\frac{1}{C_{3}} \sum_{k=0}^{n-r} \sum_{l=0}^{k}(-1)^{k}\left(\begin{array}{c}
n-r \\
k
\end{array}\right)\left(\begin{array}{l}
k \\
l
\end{array}\right) \frac{\beta\left(r_{1}+k-l+1, r_{2}+l+1\right) \Gamma\left(r_{1}+a_{1}\right) \Gamma\left(r_{2}+a_{2}+2\right)}{\left\{b_{1}+\sum_{j=1}^{r_{1}} w_{1 j}+v(k-l)\right\}^{\left(r_{1}+a_{1}\right)}\left\{b_{2}+\sum_{j=1}^{r_{2}} w_{2 j}+v(l)\right\}^{\left(r_{2}+a_{2}+2\right)}}\right]^{\frac{1}{2}}
$$

The Bayes risk of $\theta_{2}$ under PLF is obtained as: 


$$
\begin{aligned}
\mathfrak{R}\left(\theta_{2}^{*}\right)= & \left.\frac{4}{C_{3}} \sum_{k=0}^{n-r} \sum_{l=0}^{k}(-1)^{k}\left(\begin{array}{c}
n-r \\
k
\end{array}\right)\left(\begin{array}{l}
k \\
l
\end{array}\right) \frac{\beta\left(r_{1}+k-l+1, r_{2}+l+1\right) \Gamma\left(r_{1}+a_{1}\right) \Gamma\left(r_{2}+a_{2}+2\right)}{\left[b_{1}+\sum_{j=1}^{r_{1}} w_{1 j}+v(k-l)\right]^{\left(r_{1}+a_{1}\right)}\left\{b_{2}+\sum_{j=1}^{r_{2}} w_{2 j}+v(l)\right\}^{\left(r_{2}+a_{2}+2\right)}}\right]^{\frac{1}{2}} \\
& -\frac{2}{C_{3}} \sum_{k=0}^{n-r} \sum_{l=0}^{k}(-1)^{k}\left(\begin{array}{c}
n-r \\
k
\end{array}\right)\left(\begin{array}{c}
k \\
l
\end{array}\right) \frac{\beta\left(r_{1}+k-l+1, r_{2}+l+1\right) \Gamma\left(r_{1}+a_{1}\right) \Gamma\left(r_{2}+a_{2}+1\right)}{\left[b_{1}+\sum_{j=1}^{r_{1}} w_{1 j}+v(k-l)\right]^{\left(r_{1}+a_{1}\right)}\left\{b_{2}+\sum_{j=1}^{r_{2}} w_{2 j}+v(l)\right\}^{\left(r_{2}+1\right)}}
\end{aligned}
$$

The Bayes estimator of the parameter $w$ under PLF using equation (12) is obtained as:

$$
w^{*}=\left[\frac{1}{C_{3}} \sum_{k=0}^{n-r} \sum_{l=0}^{k}(-1)^{k}\left(\begin{array}{c}
n-r \\
k
\end{array}\right)\left(\begin{array}{c}
k \\
l
\end{array}\right) \frac{\beta\left(r_{1}+k+3, r_{2}+l+1\right) \Gamma\left(r_{1}+a_{1}\right) \Gamma\left(r_{2}+a_{2}\right)}{\left\{b_{1}+\sum_{j=1}^{r_{1}} w_{1 j}+v(k-l)\right\}^{\left(r_{1}+a_{1}\right)}\left\{b_{2}+\sum_{j=1}^{r_{2}} w_{2 j}+v(l)\right\}^{\left(r_{2}+a_{2}\right)}}\right]^{\frac{1}{2}}
$$

The Bayes risk of $w$ under PLF is obtained as:

$$
\begin{aligned}
& \Re\left(w^{*}\right)=\left[\frac{4}{C_{3}} \sum_{k=0}^{n-r} \sum_{l=0}^{k}(-1)^{k}\left(\begin{array}{c}
n-r \\
k
\end{array}\right)\left(\begin{array}{c}
k \\
l
\end{array}\right) \frac{\beta\left(r_{1}+k-l+3, r_{2}+l+1\right) \Gamma\left(r_{1}+a_{1}\right) \Gamma\left(r_{2}+a_{2}\right)}{\left[b_{1}+\sum_{j=1}^{r_{1}} w_{1 j}+v(k-l)\right]^{\left(r_{1}+a_{1}\right)}\left\{b_{2}+\sum_{j=1}^{r_{2}} w_{2 j}+v(l)\right\}^{\left(r_{2}+a_{2}\right)}}\right]^{\frac{1}{2}} \\
& -\frac{2}{C_{3}} \sum_{k=0}^{n-r} \sum_{l=0}^{k}(-1)^{k}\left(\begin{array}{c}
n-r \\
k
\end{array}\right)\left(\begin{array}{c}
k \\
l
\end{array}\right) \frac{\beta\left(r_{1}+k-l+2, r_{2}+l+1\right) \Gamma\left(r_{1}+a_{1}\right) \Gamma\left(r_{2}+a_{2}\right)}{\left[b_{1}+\sum_{j=1}^{r_{1}} w_{1 j}+v(k-l)\right]^{\left(r_{1}+a_{1}\right)}\left\{b_{2}+\sum_{j=1}^{r_{2}} w_{2 j}+v(l)\right\}^{\left(r_{2}+a_{2}\right)}}
\end{aligned}
$$

\section{Bayes estimators and the Bayes risks under weighted balance loss function}

The Bayes estimator of the parameter $\theta_{1}$ under WBLF using equation (10) is obtained as:

$$
=\frac{\sum_{k=0}^{n-r} \sum_{l=0}^{k}(-1)^{k}\left(\begin{array}{c}
n-r \\
k
\end{array}\right)\left(\begin{array}{l}
k \\
l
\end{array}\right) \frac{\beta\left(r_{1}+k-l+1, r_{2}+l+1\right) \Gamma\left(r_{1}+a_{1}+2\right) \Gamma\left(r_{2}+a_{2}\right)}{\left\{b_{1}+\sum_{j=1}^{r_{1}} w_{1 j}+v(k-l)\right\}^{\left(r_{1}+a_{1}+2\right)}\left\{b_{2}+\sum_{j=1}^{r_{2}} w_{2 j}+v(l)\right\}^{\left(r_{2}+a_{2}\right)}}}{\sum_{k=0}^{n-r} \sum_{l=0}^{k}(-1)^{k}\left(\begin{array}{c}
n-r \\
k
\end{array}\right)\left(\begin{array}{c}
k \\
l
\end{array}\right) \frac{\beta\left(r_{1}+k-l+1, r_{2}+l+1\right) \Gamma\left(r_{1}+a_{1}+1\right) \Gamma\left(r_{2}+a_{2}\right)}{\left\{b_{1}+\sum_{j=1}^{r_{1}} w_{1 j}+v(k-l)\right\}^{\left(r_{1}+a_{1}+1\right)}\left\{b_{2}+\sum_{j=1}^{r_{2}} w_{2 j}+v(l)\right\}^{\left(r_{2}+a_{2}\right)}}}
$$


The Bayes risk of $\theta_{1}$ under WBLF is obtained as:

$$
\mathfrak{R}\left(\theta_{1}^{*}\right)=1-\frac{\frac{1}{C_{3}}\left[\sum_{k=1}^{n-r} \sum_{l=0}^{k}(-1)^{k}\left(\begin{array}{c}
n-r \\
k
\end{array}\right)\left(\begin{array}{l}
k \\
l
\end{array}\right) \frac{\beta\left(r_{1}+k-l+1, r_{2}+l+1\right) \Gamma\left(r_{1}+a_{1}+1\right) \Gamma\left(r_{2}+a_{2}\right)}{\left\{b_{1}+\sum_{j=1}^{r_{1}} w_{1 j}+v(k-l)\right\}^{\left(r_{1}+a_{1}+1\right)}\left\{b_{2}+\sum_{j=1}^{r_{2}} w_{2 j}+v(l)\right\}^{\left(r_{2}+a_{2}\right)}}\right]^{2}}{\sum_{k=0}^{n-r} \sum_{l=0}^{k}(-1)^{k}\left(\begin{array}{c}
n-r \\
k
\end{array}\right)\left(\begin{array}{c}
k \\
l
\end{array}\right) \frac{\beta\left(r_{1}+k-l+1, r_{2}+l+1\right) \Gamma\left(r_{1}+a_{1}+2\right) \Gamma\left(r_{2}+a_{2}\right)}{\left\{b_{1}+\sum_{j=1}^{r_{1}} w_{1 j}+v(k-l)\right\}^{\left(r_{1}+a_{1}+2\right)}\left\{b_{2}+\sum_{j=1}^{r_{2}} w_{2 j}+v(l)\right\}^{\left(r_{2}+a_{2}\right)}}}
$$

The Bayes estimator of the parameter $\theta_{2}$ under WBLF using equation (11) is obtained as:

$$
=\frac{\sum_{k=0}^{n-r} \sum_{l=0}^{k}(-1)^{k}\left(\begin{array}{c}
n-r \\
k
\end{array}\right)\left(\begin{array}{l}
k \\
l
\end{array}\right) \frac{\beta\left(r_{1}+k-l+1, r_{2}+l+1\right) \Gamma\left(r_{1}+a_{1}\right) \Gamma\left(r_{2}+a_{2}+2\right)}{\left\{b_{1}+\sum_{j=1}^{r_{1}} w_{1 j}+v(k-l)\right\}^{\left(r_{1}+a_{1}\right)}\left\{b_{2}+\sum_{j=1}^{r_{2}} w_{2 j}+v(l)\right\}^{\left(r_{2}+a_{2}+2\right)}}}{\sum_{k=0}^{n-r} \sum_{l=0}^{k}(-1)^{k}\left(\begin{array}{c}
n-r \\
k
\end{array}\right)\left(\begin{array}{l}
k \\
l
\end{array}\right) \frac{\beta\left(r_{1}+k-l+1, r_{2}+l+1\right) \Gamma\left(r_{1}+a_{1}\right) \Gamma\left(r_{2}+1\right)}{\left\{b_{1}+\sum_{j=1}^{r_{1}} w_{1 j}+v(k-l)\right\}^{\left(r_{1}+a_{1}\right)}\left\{b_{2}+\sum_{j=1}^{r_{2}} w_{2 j}+v(l)\right\}^{\left(r_{2}+1\right)}}}
$$

The Bayes risk of $\theta_{2}$ under WBLF is obtained as:

$$
\mathfrak{R}\left(\theta_{2}^{*}\right)=1-\frac{\frac{1}{C_{3}}\left[\sum_{k=1}^{n-r} \sum_{l=0}^{k}(-1)^{k}\left(\begin{array}{c}
n-r \\
k
\end{array}\right)\left(\begin{array}{l}
k \\
l
\end{array}\right) \frac{\beta\left(r_{1}+k-l+1, r_{2}+l+1\right) \Gamma\left(r_{1}+a_{1}\right) \Gamma\left(r_{2}+a_{2}+1\right)}{\left\{b_{1}+\sum_{j=1}^{r_{1}} w_{1 j}+v(k-l)\right\}^{\left(r_{1}+a_{1}\right)}\left\{b_{2}+\sum_{j=1}^{r_{2}} w_{2 j}+v(l)\right\}^{\left(r_{2}+a_{2}+1\right)}}\right]^{2}}{\sum_{k=0}^{n-r} \sum_{l=0}^{k}(-1)^{k}\left(\begin{array}{c}
n-r \\
k
\end{array}\right)\left(\begin{array}{c}
k \\
l
\end{array}\right) \frac{\beta\left(r_{1}+k-l+1, r_{2}+l+1\right) \Gamma\left(r_{1}+a_{1}\right) \Gamma\left(r_{2}+a_{2}+2\right)}{\left\{b_{1}+\sum_{j=1}^{r_{1}} w_{1 j}+w(k-l)\right\}^{\left(r_{1}+a_{1}\right)}\left\{b_{2}+\sum_{j=1}^{r_{2}} w_{2 j}+v(l)\right\}^{\left(r_{2}+a_{2}+2\right)}}}
$$

The Bayes estimator of the parameter $w$ under WBLF using equation (12) is obtained as:

$$
=\frac{\sum_{k=0}^{n-r} \sum_{l=0}^{k}(-1)^{k}\left(\begin{array}{c}
n-r \\
k
\end{array}\right)\left(\begin{array}{l}
k \\
l
\end{array}\right) \frac{\beta\left(r_{1}+k-l+3, r_{2}+l+1\right) \Gamma\left(r_{1}+a_{1}\right) \Gamma\left(r_{2}+a_{2}\right)}{\left\{b_{1}+\sum_{j=1}^{r_{1}} w_{1 j}+v(k-l)\right\}^{\left(r_{1}+a_{1}\right)}\left\{b_{2}+\sum_{j=1}^{r_{2}} w_{2 j}+v(l)\right\}^{\left(r_{2}+a_{2}\right)}}}{\sum_{k=0}^{n-r} \sum_{l=0}^{k}(-1)^{k}\left(\begin{array}{c}
n-r \\
k
\end{array}\right)\left(\begin{array}{l}
k \\
l
\end{array}\right) \frac{\beta\left(r_{1}+k-l+2, r_{2}+l+1\right) \Gamma\left(r_{1}+a_{1}\right) \Gamma\left(r_{2}+a_{2}\right)}{\left\{b_{1}+\sum_{j=1}^{r_{1}} w_{1 j}+v(k-l)\right\}^{\left(r_{1}+a_{1}\right)}\left\{b_{2}+\sum_{j=1}^{r_{2}} w_{2 j}+v(l)\right\}^{\left(r_{2}+a_{2}\right)}}}
$$


The Bayes risk of $w$ under WBLF is obtained as:

$\mathfrak{R}\left(w^{*}\right)=1-\frac{\frac{1}{C_{3}}\left[\sum_{k=1}^{n-r} \sum_{l=0}^{k}(-1)^{k}\left(\begin{array}{c}n-r \\ k\end{array}\right)\left(\begin{array}{c}k \\ l\end{array}\right) \frac{\beta\left(r_{1}+k-l+2, r_{2}+l+1\right) \Gamma\left(r_{1}+a_{1}\right) \Gamma\left(r_{2}+a_{2}\right)}{\left\{b_{1}+\sum_{j=1}^{r_{1}} w_{1 j}+v(k-l)\right\}^{\left(r_{1}+a_{1}\right)}\left\{b_{2}+\sum_{j=1}^{r_{2}} w_{2 j}+v(l)\right\}^{\left(r_{2}+a_{2}\right)}}\right]^{2}}{\sum_{k=0}^{n-r} \sum_{l=0}^{k}(-1)^{k}\left(\begin{array}{c}n-r \\ k\end{array}\right)\left(\begin{array}{c}k \\ l\end{array}\right) \frac{\beta\left(r_{1}+k-l+3, r_{2}+l+1\right) \Gamma\left(r_{1}+a_{1}\right) \Gamma\left(r_{2}+a_{2}\right)}{\left\{b_{1}+\sum_{j=1}^{r_{1}} w_{1 j}+v(k-l)\right\}^{\left(r_{1}+a_{1}\right)}\left\{b_{2}+\sum_{j=1}^{r_{2}} w_{2 j}+v(l)\right\}^{\left(r_{2}+a_{2}\right)}}}$

\section{Bayes estimators and the Bayes risks under weighted loss function}

The Bayes estimator of the parameter $\theta_{1}$ under WLF using equation (10) is obtained as:

$\theta_{1}^{*}=\left[\frac{1}{C_{3}} \sum_{k=0}^{n-r} \sum_{l=0}^{k}(-1)^{k}\left(\begin{array}{c}n-r \\ k\end{array}\right)\left(\begin{array}{l}k \\ l\end{array}\right) \frac{\beta\left(r_{1}+k-l+1, r_{2}+l+1\right) \Gamma\left(r_{2}+a_{2}\right) \Gamma\left(r_{1}+a_{1}-1\right)}{\left\{\sum_{j=1}^{r_{2}} w_{2 j}+v(l)\right\}^{\left(r_{2}+a_{2}\right)}\left\{\sum_{j=1}^{r_{1}} w_{1 j}+v(k-l)\right\}^{\left(r_{1}+a_{1}-1\right)}}\right]^{-1}$

The Bayes risk of $\theta_{1}$ under WLF is obtained as:

$$
\mathfrak{R}\left(\theta_{1}^{*}\right)=\frac{1}{C_{3}} \sum_{k=0}^{n-r} \sum_{l=0}^{k}(-1)^{k}\left(\begin{array}{c}
n-r \\
k
\end{array}\right)\left(\begin{array}{c}
k \\
l
\end{array}\right) \frac{\beta\left(r_{1}+k-l+1, r_{2}+l+1\right) \Gamma\left(r_{2,}+a_{2}\right) \Gamma\left(r_{1}+a_{1}+1\right)}{\left\{\sum_{j=1}^{r_{2}} w_{2 j}+v(l)\right\}^{\left(r_{2}+a_{2}\right)}\left\{\sum_{j=1}^{r_{1}} w_{1 j}+v(k-l)\right\}^{\left(r_{1}+a_{1}+1\right)}}
$$

The Bayes estimator of the parameter $\theta_{2}$ under WLF using equation (11) is obtained as:

$$
\theta_{2}^{*}=\left[\frac{1}{C_{3}} \sum_{k=0}^{n-r} \sum_{l=0}^{k}(-1)^{k}\left(\begin{array}{c}
n-r \\
k
\end{array}\right)\left(\begin{array}{c}
k \\
l
\end{array}\right) \frac{\beta\left(r_{1}+k-l+1, r_{2}+l+1\right) \Gamma\left(r_{2}+a_{2}-1\right) \Gamma\left(r_{1}+a_{1}\right)}{\left\{\sum_{j=1}^{r_{2}} w_{2 j}+v(l)\right\}^{\left(r_{2}+a_{2}-1\right)}\left\{\sum_{j=1}^{r_{1}} w_{1 j}+v(k-l)\right\}^{\left(r_{1}+a_{1}\right)}}\right]^{-1}
$$

The Bayes risk of $\theta_{2}$ under WLF is obtained as:

$$
\begin{aligned}
\mathfrak{R}\left(\theta_{2}^{*}\right) & =\frac{1}{C_{3}} \sum_{k=0}^{n-r} \sum_{l=0}^{k}(-1)^{k}\left(\begin{array}{c}
n-r \\
k
\end{array}\right)\left(\begin{array}{c}
k \\
l
\end{array}\right) \frac{\beta\left(r_{1}+k-l+1, r_{2}+l+1\right) \Gamma\left(r_{2,}+a_{2}+1\right) \Gamma\left(r_{1}+a_{1}\right)}{\left\{\sum_{j=1}^{r_{2}} w_{2 j}+v(l)\right\}^{\left(r_{2}+a_{2}+1\right)}\left\{\sum_{j=1}^{r_{1}} w_{1 j}+v(k-l)\right\}^{\left(r_{1}+a_{1}\right)}} \\
& -\left[\frac{1}{C_{3}} \sum_{k=0}^{n-r} \sum_{l=0}^{k}(-1)^{k}\left(\begin{array}{c}
n-r \\
k
\end{array}\right)\left(\begin{array}{c}
k \\
l
\end{array}\right) \frac{\beta\left(r_{1}+k-l+1, r_{2}+l+1\right) \Gamma\left(r_{2}+a_{2}-1\right) \Gamma\left(r_{1}+a_{1}\right)}{\left\{\sum_{j=1}^{r_{2}} w_{2 j}+v(l)\right\}^{\left(r_{2}+a_{2}-1\right)}\left\{\sum_{j=1}^{r_{1}} w_{1 j}+v(k-l)\right\}^{\left(r_{1}+a_{1}\right)}}\right]^{-1}
\end{aligned}
$$


The Bayes estimator of the parameter $w$ under WLF using equation (12) is obtained as:

$$
w^{*}=\left[\frac{1}{C_{3}} \sum_{k=0}^{n-r} \sum_{l=0}^{k}(-1)^{k}\left(\begin{array}{c}
n-r \\
k
\end{array}\right)\left(\begin{array}{c}
k \\
l
\end{array}\right) \frac{\beta\left(r_{1}+k-l, r_{2}+l+1\right) \Gamma\left(r_{2,}+a_{2}\right) \Gamma\left(r_{1}+a_{1}\right)}{\left\{\sum_{j=1}^{r_{2}} w_{2 j}+v(l)\right\}^{\left(r_{2}+a_{2}\right)}\left\{\sum_{j=1}^{r_{1}} w_{1 j}+v(k-l)\right\}^{\left(r_{1}+a_{1}\right)}}\right]^{-1}
$$

The Bayes risk of $w$ under WLF is obtained as:

$$
\begin{aligned}
\Re\left(w^{*}\right)= & \frac{1}{C_{3}} \sum_{k=0}^{n-r} \sum_{l=0}^{k}(-1)^{k}\left(\begin{array}{c}
n-r \\
k
\end{array}\right)\left(\begin{array}{l}
k \\
l
\end{array}\right) \frac{\beta\left(r_{1}+k-l+2, r_{2}+l+1\right) \Gamma\left(r_{2,}\right) \Gamma\left(r_{1}\right)}{\left\{\sum_{j=1}^{r_{2}} w_{2 j}+v(l)\right\}^{\left(r_{2}\right)}\left\{\sum_{j=1}^{r_{1}} w_{1 j}+v(k-l)\right\}^{\left(r_{1}\right)}} \\
& -\left[\frac{1}{C_{3}} \sum_{k=0}^{n-r} \sum_{l=0}^{k}(-1)^{k}\left(\begin{array}{c}
n-r \\
k
\end{array}\right)\left(\begin{array}{c}
k \\
l
\end{array}\right) \frac{\beta\left(r_{1}+k-l, r_{2}+l+1\right) \Gamma\left(r_{2,}+a_{2}\right) \Gamma\left(r_{1}+a_{1}\right)}{\left\{\sum_{j=1}^{r_{2}} w_{2 j}+v(l)\right\}^{\left(r_{2}+a_{2}\right)}\left\{\sum_{j=1}^{r_{1}} w_{1 j}+v(k-l)\right\}^{\left(r_{1}+a_{1}\right)}}\right]^{-1}
\end{aligned}
$$

\section{Posterior distribution assuming inverse Levy prior}

In addition to the GP, inverse Levy prior (ILP) is taken as an informative prior. It is defined as: $p\left(\theta_{1}\right)=\left(\frac{b_{1}}{2 \pi}\right)^{\frac{1}{2}} \theta_{1}^{-\frac{1}{2}} e^{-\frac{b_{1}}{2} \theta_{1}}$; $\theta_{1}>0, \quad p\left(\theta_{2}\right)=\left(\frac{b_{2}}{2 \pi}\right)^{\frac{1}{2}} \theta_{2}^{-\frac{1}{2}} e^{-\frac{b_{2}}{2} \theta_{2}}, \quad \theta_{2}>0$ whereas $p(w)=1 ; \quad 0<w<1$, Thus, the joint prior distribution of $\theta_{1}, \theta_{2}$ and $w$ is given by:

$$
\begin{aligned}
& p\left(\theta_{1}, \theta_{2}, w\right) \propto \theta_{1}^{-\frac{1}{2}} \theta_{2}^{-\frac{1}{2}} e^{-\frac{b_{1}}{2} \theta_{1}-\frac{b_{2}}{2} \theta_{2}} ; \\
& \theta_{1}, \theta_{2}>0, \quad 0<w<1
\end{aligned}
$$

The joint posterior distribution of $\theta_{1}, \theta_{2}$ and $w$ is given below:

$$
\begin{aligned}
& p\left(\theta_{1}, \theta_{2}, w \mid x\right) \propto \sum_{k=0}^{n-r} \sum_{l=0}^{k}(-1)^{k}\left(\begin{array}{c}
n-r \\
k
\end{array}\right)\left(\begin{array}{l}
k \\
l
\end{array}\right) \\
& \theta_{1}^{r_{1}-\frac{1}{2}} \theta_{2}^{r_{2}-\frac{1}{2}} w^{r_{1}+k-l}(1-w)^{r_{2}+l} \\
& \times \exp \left[-\theta_{1}\left\{\frac{b_{1}}{2}+\sum_{j=1}^{r_{1}} w_{1 j}+v(k-l)\right\}-\theta_{2}\left\{\frac{b_{2}}{2}+\sum_{j=1}^{r_{2}} w_{2 j}+v(l)\right\}\right] \\
& \theta_{1}, \theta_{2}>0,0<w<1
\end{aligned}
$$

The marginal posterior distribution of $\theta_{1}$ can be written as:

$$
\begin{aligned}
& p\left(\theta_{1} \mid x\right)= \frac{1}{C_{4}} \sum_{k=0}^{n-r} \sum_{l=0}^{k}(-1)^{k}\left(\begin{array}{c}
n-r \\
k
\end{array}\right)\left(\begin{array}{l}
k \\
l
\end{array}\right) \beta\left(r_{1}+k-l+1, r_{2}+l+1\right) \\
& \times \frac{\Gamma\left(r_{2}+\frac{1}{2}\right)}{\left\{\frac{b_{2}}{2}+\sum_{j=1}^{r_{2}} w_{2 j}+v(l)\right\}^{\left(r_{2}+\frac{1}{2}\right)}} \\
& \theta_{1}^{r_{1}-\frac{1}{2}} \exp \left[-\theta_{1}\left\{\frac{b_{1}}{2}+\sum_{j=1}^{r_{1}} w_{1 j}+v(k-l)\right\}\right] ; \theta_{1}>0
\end{aligned}
$$

where $C_{4}=\int_{0}^{\infty} \int_{0}^{\infty} \int_{0}^{1} \sum_{k=0}^{n-r} \sum_{l=0}^{k}(-1)^{k}\left(\begin{array}{c}n-r \\ k\end{array}\right)\left(\begin{array}{c}k \\ l\end{array}\right)$

$$
\begin{aligned}
& \theta_{1}^{r_{1}-\frac{1}{2}} \theta_{2}^{r_{2}-\frac{1}{2}} w^{r_{1}+k-l}(1-w)^{r_{2}+l} \\
& \times \exp \left[-\theta_{1}\left\{\frac{b_{1}}{2}+\sum_{j=1}^{r_{1}} w_{1 j}+(k-l) v\right\}-\right. \\
& \left.\theta_{2}\left\{\frac{b_{2}}{2}+\sum_{j=1}^{r_{2}} w_{2 j}+v(l)\right\}\right] d w d \theta_{2} d \theta_{1}
\end{aligned}
$$




$$
\begin{aligned}
& =\sum_{k=0}^{n-r} \sum_{l=0}^{k}(-1)^{k}\left(\begin{array}{c}
n-r \\
k
\end{array}\right)\left(\begin{array}{l}
k \\
l
\end{array}\right) \beta\left(r_{1}+k-l+1, r_{2}+l+1\right) \\
& \times \frac{\Gamma\left(r_{1}+\frac{1}{2}\right) \Gamma\left(r_{2}+\frac{1}{2}\right)}{\left\{\frac{b_{1}}{2}+\sum_{j=1}^{r_{1}} w_{1 j}+v(k-l)\right\}^{\left(r_{1}+\frac{1}{2}\right)}\left\{\frac{b_{2}}{2}+\sum_{j=1}^{r_{2}} w_{2 j}+v(l)\right\}^{\left(r_{2}+\frac{1}{2}\right)}}
\end{aligned}
$$

The marginal posterior distribution of $\theta_{2}$ is obtained as:

$$
\begin{aligned}
p\left(\theta_{1} \mid x\right)= & \frac{1}{C_{4}} \sum_{k=0}^{n-r} \sum_{l=0}^{k}(-1)^{k}\left(\begin{array}{c}
n-r \\
k
\end{array}\right)\left(\begin{array}{l}
k \\
l
\end{array}\right) \beta\left(r_{1}+k-l+1, r_{2}+l+1\right) \\
& \times \frac{\Gamma\left(r_{1}+\frac{1}{2}\right)}{\left\{\frac{b_{1}}{2}+\sum_{j=1}^{r_{1}} w_{1 j}+v(k-l)\right\}^{\left(r_{1}+\frac{1}{2}\right)}} \theta_{1}^{r_{2}-\frac{1}{2}} \\
& \exp \left[-\theta_{2}\left\{\frac{b_{2}}{2}+\sum_{j=1}^{r_{2}} w_{2 j}+v(l)\right\}\right]
\end{aligned}
$$

The marginal posterior distribution of $w$ is obtained as:

$$
\begin{aligned}
& p(w \mid x)=\frac{1}{C_{4}} \sum_{k=0}^{n-r} \sum_{l=0}^{k}(-1)^{k}\left(\begin{array}{c}
n-r \\
k
\end{array}\right)\left(\begin{array}{l}
k \\
l
\end{array}\right) \\
& \times \frac{\Gamma\left(r_{1}+\frac{1}{2}\right) \Gamma\left(r_{2}+\frac{1}{2}\right) w^{r_{1}+k-l}(1-w)^{r_{2}+l}}{\left\{\frac{b_{1}}{2}+\sum_{j=1}^{r_{1}} w_{1 j}+v(k-l)\right\}^{\left(r_{1}+\frac{1}{2}\right)}\left\{\frac{b_{2}}{2}+\sum_{j=1}^{r_{2}} w_{2 j}+v(l)\right\}^{\left(r_{2}+\frac{1}{2}\right)}}
\end{aligned}
$$

$0<w<1$

Assuming $\quad a_{1}=a_{2}=0.5$, and $b_{1}=b_{1} / 2, b_{2}=b_{2} / 2$ in equations (13) to (42), one can obtain the Bayes estimators and their respective posterior risk under different loss functions for the JP.

\section{Posterior distribution under noninformative priors}

In this section, the derivation of the posterior distributions assuming noninformative priors is discussed. Noninformative priors are priors which are less informative than informative priors. The two most commonly used noninformative priors are the Jeffreys prior (JP) and uniform prior (UP) (Ali et al., 2012).

$\boldsymbol{J P}:$ The Jeffreys prior is given by $p\left(\theta_{1}\right) \propto \frac{1}{\theta_{1}} ; \theta_{1}>0$, $p\left(\theta_{2}\right) \propto \frac{1}{\theta_{2}} ; \theta_{2}>0$, while the uniform prior is assumed for $w$, i.e., $p(w)=1 ; 0<w<1$, then the joint prior distribution of $\theta_{1}, \theta_{2}$ and $w$ is:

$$
p\left(\theta_{1}, \theta_{2}, w\right) \propto \frac{1}{\theta_{1} \theta_{2}} ; \theta_{1}, \theta_{2}>0,0<w<1,
$$

Thus, the joint posterior distribution of $\theta_{1}, \theta_{2}$ and $w$ is given by:

$$
\begin{aligned}
& p\left(\theta_{1}, \theta_{2}, w \mid \mathbf{x}\right) \propto \sum_{k=0}^{n-r} \sum_{l=0}^{k}(-1)^{k}\left(\begin{array}{c}
n-r \\
k
\end{array}\right)\left(\begin{array}{l}
k \\
l
\end{array}\right) \\
& \mid \theta_{1}^{r_{1}-1} \theta_{2}^{r_{2}-1} w^{r_{1}+k-l}(1-w)^{r_{2}+l} \times \exp \left[-\theta_{1}\left\{\sum_{j=1}^{r_{1}} w_{1 j}+v(k-l)\right\}\right. \\
& \left.-\theta_{2}\left\{\sum_{j=1}^{r_{2}} w_{2 j}+v(l)\right\}\right], \theta_{1}, \theta_{2}>0,0<w<1
\end{aligned}
$$$$
p\left(\theta_{1}, \theta_{2}, w \mid \mathbf{x}\right)=\frac{1}{C_{2}} \sum_{k=0}^{n-r} \sum_{l=0}^{k}(-1)^{k}\left(\begin{array}{c}
n-r \\
k
\end{array}\right)\left(\begin{array}{l}
k \\
l
\end{array}\right)
$$$$
\theta_{1}^{r_{1}-1} \theta_{2}^{r_{2}-1} w^{r_{1}+k-l}(1-w)^{r_{2}+l} \times \exp \left[-\theta_{1}\left\{\sum_{j=1}^{r_{1}} w_{1 j}+v(k-l)\right\}\right.
$$$$
\left.-\theta_{2}\left\{\sum_{j=1}^{r_{2}} w_{2 j}+v(l)\right\}\right], \theta_{1}, \theta_{2}>0,0<w<1
$$

where $\mathrm{C}_{2}=\sum_{k=0}^{n-r} \sum_{l=0}^{k}(-1)^{k}\left(\begin{array}{c}n-r \\ k\end{array}\right)\left(\begin{array}{l}k \\ l\end{array}\right)$

$\beta\left(r_{1}+k-l+1, r_{2}+l+1\right)^{*}$

$$
\frac{\Gamma\left(r_{1}\right) \Gamma\left(r_{2}\right)}{\left\{\sum_{j=1}^{r_{1}} w_{1 j}+v(k-l)\right\}^{\left(r_{1}\right)}\left\{\sum_{j=1}^{r_{2}} w_{2 j}+v(l)\right\}^{\left(r_{2}\right)}}
$$

The marginal posterior distribution of $\theta_{1}$ using JP is obtained from equation (49) and is given by: 


$$
\begin{aligned}
& p\left(\theta_{1} \mid \mathbf{x}\right)=\frac{1}{C_{2}} \sum_{k=0}^{n-r} \sum_{l=0}^{k}(-1)^{k}\left(\begin{array}{c}
n-r \\
k
\end{array}\right)\left(\begin{array}{l}
k \\
l
\end{array}\right) \\
& \beta\left(r_{1}+k-l+1, r_{2}+l+1\right) \\
& \times \frac{\Gamma\left(r_{2}\right)}{\left\{\sum_{j=1}^{r_{1}} w_{2 j}+v(l)\right\}^{r_{2}}} \theta_{1}^{r_{1}-1} \exp \left[-\theta_{1}\left\{\sum_{j=1}^{r_{2}} w_{1 j}+v(k-l)\right\} ; \theta_{1}>0\right.
\end{aligned}
$$

The marginal posterior distribution of $\theta_{2}$ is given by:

$$
\begin{aligned}
& p\left(\theta_{2} \mid \mathbf{x}\right)=\frac{1}{C_{2}} \sum_{k=0}^{n-r} \sum_{l=0}^{k}(-1)^{k}\left(\begin{array}{c}
n-r \\
k
\end{array}\right)\left(\begin{array}{l}
k \\
l
\end{array}\right) \\
& \beta\left(r_{1}+k-l+1, r_{2}+l+1\right) \\
& \times \frac{\Gamma\left(r_{1}\right)}{\left\{\sum_{j=1}^{r_{1}} w_{1 j}+v(k-l)\right\}^{\left(r_{1}\right)}} \theta_{2}^{r_{2}-1} \exp \left[-\theta_{2}\left\{\sum_{j=1}^{r_{2}} w_{2 j}+v(l)\right\}\right] \cdot \theta_{2}>0
\end{aligned}
$$

The marginal posterior distribution of $w$ is given below:

$$
\begin{aligned}
& p(w \mid \mathbf{x})=\frac{1}{C_{2}} \sum_{k=0}^{n-r} \sum_{l=0}^{k}(-1)^{k}\left(\begin{array}{c}
n-r \\
k
\end{array}\right)\left(\begin{array}{l}
k \\
l
\end{array}\right) \\
& \times \frac{\Gamma\left(r_{1}\right) \Gamma\left(r_{2}\right) w^{r_{1}+k-l}(1-w)^{r_{2}+l}}{\left\{\sum_{j=1}^{r_{1}} w_{1 j}+v(k-l)\right\}^{\left(r_{1}\right)}\left\{\sum_{j=1}^{r_{2}} w_{2 j}+v(l)\right\}^{\left(r_{2}\right)}} 0<w<1
\end{aligned}
$$

Assuming $a_{1}=a_{2} \rightarrow 0$ and $b_{1}=b_{2} \rightarrow 0$ in equations (13) to (42), the Bayes estimators and the respective posterior risks under different loss functions for the JP can be obtained.

$\boldsymbol{U P}$ : Now, considering the uniform prior, i.e., $\theta_{1}, \theta_{2} \sim$ $U(0, \infty)$ and $w \sim U(0,1)$, the joint prior distribution of $\theta_{1}, \theta_{2}$ and $w$ is given by:

$p\left(\theta_{1}, \theta_{2}, w\right) \alpha 1 ; \quad \theta_{1}, \theta_{2}>0, \quad 0<w<1$

The joint posterior distribution of $\theta_{1}, \theta_{2}$ and $w$ is given by:

$$
\begin{aligned}
& p\left(\theta_{1}, \theta_{2}, w \mid \mathbf{x}\right) \propto \sum_{k=0}^{n-r} \sum_{l=0}^{k}(-1)^{k}\left(\begin{array}{c}
n-r \\
k
\end{array}\right)\left(\begin{array}{l}
k \\
l
\end{array}\right) \\
& \theta_{1}^{r_{1}} \theta_{2}^{r_{2}} w^{r_{1}+k-l}(1-w)^{r_{2}+l} \times \exp \left[-\theta_{1}\left\{\sum_{j=1}^{r_{1}} w_{1 j}+v(k-l)\right\}\right. \\
& \left.-\theta_{2}\left\{\sum_{j=1}^{r_{2}} w_{2 j}+v(l)\right\}\right], \theta_{1}, \theta_{2}>0,0<w<1
\end{aligned}
$$$$
p\left(\theta_{1}, \theta_{2}, w \mid \mathbf{x}\right)=\frac{1}{C_{1}} \sum_{k=0}^{n-r} \sum_{l=0}^{k}(-1)^{k}\left(\begin{array}{c}
n-r \\
k
\end{array}\right)\left(\begin{array}{l}
k \\
l
\end{array}\right)
$$$$
\theta_{1}^{r_{1}} \theta_{2}^{r_{2}} w^{r_{1}+k-l}(1-w)^{r_{2}+l} \times \exp \left[-\theta_{1}\left\{\sum_{j=1}^{r_{1}} w_{1 j}+v(k-l)\right\}\right.
$$$$
\left.-\theta_{2}\left\{\sum_{j=1}^{r_{2}} w_{2 j}+v(l)\right\}\right], \theta_{1}, \theta_{2}>0,0<w<1,
$$

where

$$
C_{1}=\sum_{k=0}^{n-r} \sum_{l=0}^{k}(-1)^{k}\left(\begin{array}{c}
n-r \\
k
\end{array}\right)\left(\begin{array}{l}
k \\
l
\end{array}\right)
$$

$\beta\left(r_{1}+k-l+1, r_{2}+l+1\right)$

$* \frac{\Gamma\left(r_{1}+1\right) \Gamma\left(r_{2}+1\right)}{\left\{\sum_{j=1}^{r_{1}} w_{1 j}+v(k-l)\right\}^{\left(r_{1}+1\right)}\left\{\sum_{j=1}^{r_{2}} w_{2 j}+v(l)\right\}^{\left(r_{2}+1\right)}}$

The marginal posterior distribution of $\theta_{1}$ using the UP is obtained from equation (54):

$$
p\left(\theta_{1} \mid \mathbf{x}\right)=\frac{1}{C_{1}} \sum_{k=0}^{n-r} \sum_{l=0}^{k}(-1)^{k}\left(\begin{array}{c}
n-r \\
k
\end{array}\right)\left(\begin{array}{l}
k \\
l
\end{array}\right)
$$

$$
\beta\left(r_{1}+k-l+1, r_{2}+l+1\right) \frac{\Gamma\left(r_{2}+1\right)}{\left\{\sum_{j=1}^{r_{2}} w_{2 j}+v(l)\right\}^{\left(r_{2}+1\right)}} \theta_{1}^{r_{1}}
$$

$\exp \left[-\theta_{1}\left\{\sum_{j=1}^{r_{1}} w_{1 j}+v(k-l)\right\}^{\left(r_{1}+1\right)}\right] ; \theta_{1}>0$ 
The marginal posterior distribution of $\theta_{2}$ is given by:

$$
\begin{aligned}
& p\left(\theta_{2} \mid x\right)=\frac{1}{C_{1}} \sum_{k=0}^{n-r} \sum_{l=0}^{k}(-1)^{k}\left(\begin{array}{c}
n-r \\
k
\end{array}\right)\left(\begin{array}{l}
k \\
l
\end{array}\right) \\
& \beta\left(r_{1}+k-l+1, r_{2}+l+1\right) \times \frac{\Gamma\left(r_{1}+1\right)}{\left\{\sum_{j=1}^{r_{1}} w_{1 j}+v(k-l)\right\}^{\left(r_{1}+1\right)}} \theta_{2}^{r_{2}} \\
& \exp \left[-\theta_{2}\left\{\sum_{j=1}^{r_{2}} w_{2 j}+v(l)\right\}\right] ; \theta_{2}>0
\end{aligned}
$$

The marginal posterior distribution of $w$ is obtained as:

$$
\begin{aligned}
& p(w \mid \mathbf{x})=\frac{1}{C_{1}} \sum_{k=0}^{n-r} \sum_{l=0}^{k}(-1)^{k}\left(\begin{array}{c}
n-r \\
k
\end{array}\right)\left(\begin{array}{l}
k \\
l
\end{array}\right) \\
& \times \frac{\Gamma\left(r_{1}+1\right) \Gamma\left(r_{2}+1\right) w^{r_{1}+k-l}(1-w)^{r_{2}+l}}{\left\{\sum_{j=1}^{r_{1}} w_{1 j}+v(k-l)\right\}^{\left(r_{1}+1\right)}\left\{\sum_{j=1}^{r_{2}} w_{2 j}+v(l)\right\}^{\left(r_{2}+1\right)},} \\
& 0<w<1 .
\end{aligned}
$$

Assuming $a_{1}=a_{2}=1$ and $b_{1}=b_{2} \rightarrow 0$ in equations (13) to
(42), the Bayes estimators and their respective posterior risks under different loss functions for the UP can be obtained.

\section{RESULTS AND DISCUSSION}

\section{Real life data application}

Murthy et al. (2004) presented a real-life dataset, which is given in Table 2, consisting of 88 observations on the failure times of windshields. The descriptive summary of the data is given in Table 3 while the Bayes estimates and their posterior risks under different loss functions are given in Tables 4 and 5.

Table 2: Real life dataset

\begin{tabular}{llllllll}
\hline 0.040 & 0.301 & 0.309 & 0.557 & 0.943 & 1.070 & 1.124 & 1.248 \\
1.281 & 1.281 & 1.303 & 1.432 & 1.480 & 1.505 & 1.506 & 1.568 \\
1.615 & 1.619 & 1.652 & 1.652 & 1.757 & 1.795 & 1.866 & 1.876 \\
1.899 & 1.911 & 1.912 & 1.914 & 1.981 & 2.010 & 2.038 & 2.085 \\
2.085 & 2.097 & 2.135 & 2.154 & 2.190 & 2.194 & 2.223 & 2.224 \\
2.229 & 2.300 & 2.324 & 2.349 & 2.385 & 2.481 & 2.610 & 2.625 \\
2.632 & 2.646 & 2.661 & 2.688 & 2.823 & 2.890 & 2.902 & 2.934 \\
2.962 & 2.964 & 3.000 & 3.103 & 3.114 & 3.117 & 3.166 & 3.344 \\
3.376 & 3.385 & 3.443 & 3.467 & 3.478 & 3.578 & 3.595 & 3.699 \\
3.779 & 3.924 & 4.035 & 4.121 & 4.167 & 4.240 & 4.255 & 4.278 \\
4.305 & 4.376 & 4.449 & 4.485 & 4.570 & 4.602 & 4.663 & 4.694 \\
\hline & & & & & & &
\end{tabular}

Table 3: Descriptive summary of real data

\begin{tabular}{cccccccccc}
\hline $\mathrm{n}$ & Mean & $\mathrm{SD}$ & $\mathrm{SE}$ & Mode & Median & Kurtosis & Skewness & Minimum & Maximum \\
88 & 2.58 & 1.12 & 0.12 & 1.28 & 2.36 & -0.62 & 0.12 & 0.04 & 4.69 \\
\hline
\end{tabular}

Table 4: BEs and BRs for the real dataset using non-informative priors and $20 \%$ censoring rate

\begin{tabular}{lcccc}
\hline $\begin{array}{l}\text { Loss } \\
\text { functions }\end{array}$ & BEs $\left(\theta_{1}, \theta_{2}, \beta=1, w\right)$ & PRs & BEs $\left(\theta_{1}, \theta_{2}, \beta=1, w\right)$ & Peffreys prior \\
\hline SELF & $1.6398,2.0559,0.3875$ & $0.0844,0.0952,0.0035$ & $1.5897,2.0112,0.3867$ & $0.0819,0.0932,0.0035$ \\
QLF & $1.5368,1.9631,0.3784$ & $0.0325,0.0231,0.0237$ & $1.4864,1.9183,0.3681$ & $0.9664,0.0236,0.0249$ \\
PLF & $1.6654,2.0789,0.3852$ & $0.0510,0.0461,0.0237$ & $1.6152,2.0342,0.3781$ & $0.0511,0.0461,0.0242$ \\
WLF & $1.5719,2.0088,0.3920$ & $0.0304,0.0251,0.0089$ & $1.5695,1.9855,0.3911$ & $0.9701,0.0292,0.0089$ \\
WBLF & $1.6913,2.1022,0.3965$ & $0.0304,0.0220,0.0226$ & $1.6412,2.0575,0.3957$ & $0.0314,0.0225,0.0227$ \\
\hline
\end{tabular}


This dataset is considered because failure times follow the Weibull distribution (Murthy et al., 2004) and EIWD is a more flexible generalisation of the ordinary Weibull distribution. Thus, it is more appropriate to use the dataset for further analysis. Using the above dataset,
BEs and PRs have been calculated under five different loss functions (SELF, QLF, PLF, WLF and WBLF) and different types of non-informative and informative priors. The resulting study is tabulated in Tables 4 and 5 while the graphical depiction is given in Figure 2.

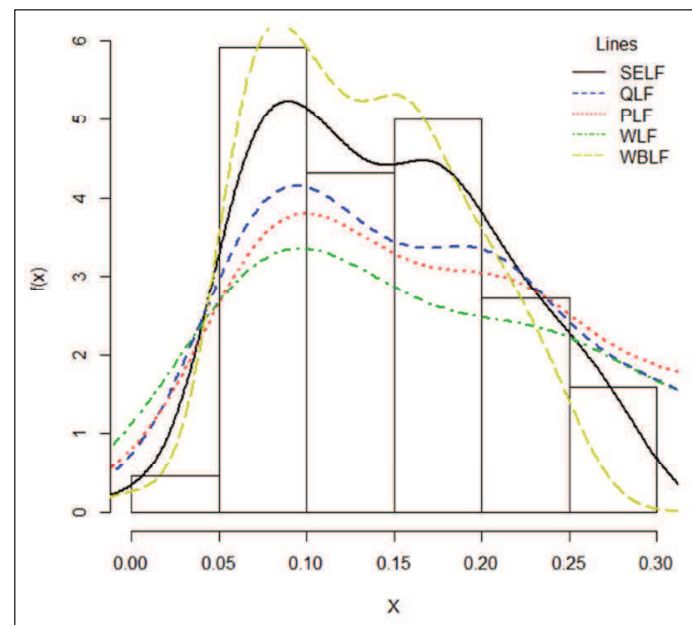

(a) Uniform prior

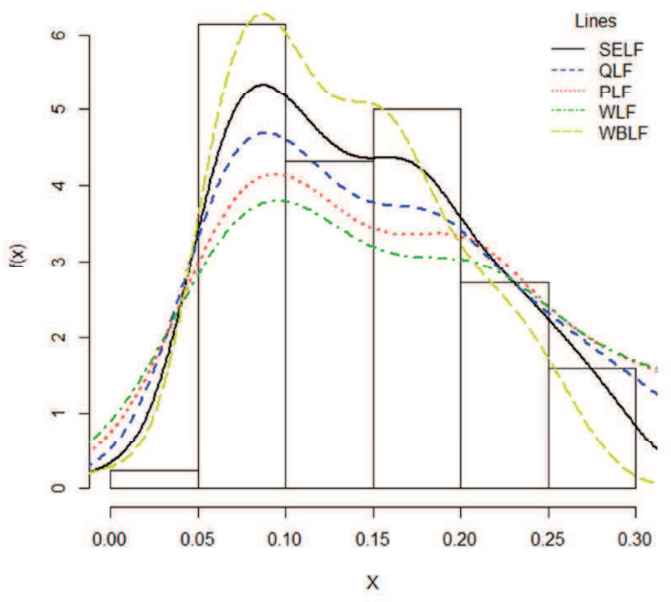

(c) Gamma prior

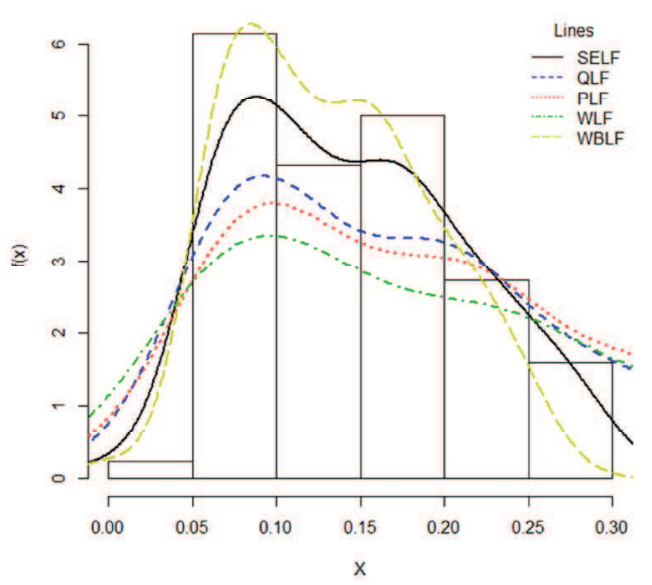

(b) Jeffreys prior

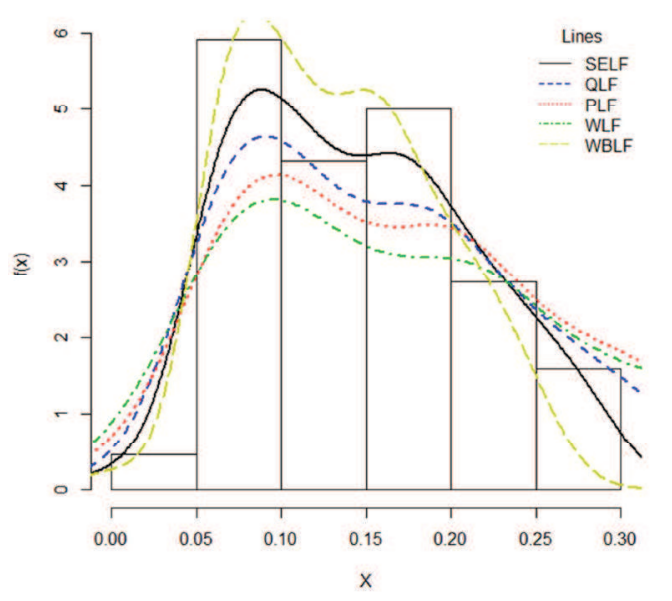

(d) Inverse Levy prior

Figure 2: A comparison of observed and theoretical densities using UP, JP, GP and ILP

From Table 4, by comparing Bayes risks of different loss functions, it is obvious that WBLF has the minimum posterior risk as compared to the other loss functions. Therefore, the WBLF is the most suitable loss function for the Bayesian estimation of the scale parameter of the EIWD. The above results also suggest that the performance of the uniform prior is better than the Jeffreys prior. Similar to Table 4, Table 5 suggests that the gamma prior is better than the inverse Levy prior and WLF has the minimum risk as compared to the rest. It is also observed that informative priors are more preferable than the noninformative priors because of minimum risks, and gamma prior has the least risk among the assumed priors. It is worth mentioning that the hyperparameters of the informative priors are selected to represent mild information than the noninformative case. A graphical 
depiction of the observed and theoretical densities have been given in Figure 2. The interpretation of Figure 2 is the same as the interpretation of the results given in Tables 4 and 5 .

Table 5: BEs and BRs for the real dataset using informative priors and $20 \%$ censoring rate

\begin{tabular}{ccccc}
\hline $\begin{array}{c}\text { Loss } \\
\text { functions }\end{array}$ & BEs $\left(\theta_{1}, \theta_{2}, \beta=1, w\right)$ & PRs & BEs $\left(\theta_{1}, \theta_{2}, \beta=1, w\right)$ & PRs \\
\hline SELF & $1.5254,2.0112,0.3853$ & $0.0721,0.0932,0.0035$ & $1.6041,2.0309,0.3869$ & $0.0820,0.0939,0.0035$ \\
QLF & $1.4307,1.9183,0.3667$ & $0.0279,0.0236,0.0249$ & $1.5017,1.9382,0.3684$ & $0.0330,0.9766,0.0248$ \\
PLF & $1.5489,1.9513,0.3898$ & $0.0469,0.0426,0.0089$ & $1.6295,2.0539,0.3915$ & $0.0507,0.0460,0.0089$ \\
WLF & $1.5074,1.9325,0.3745$ & $0.0234,0.0287,0.0255$ & $1.5156,1.9728,0.3651$ & $0.0321,0.9663,0.0237$ \\
WBLF & $1.5727,1.9729,0.3943$ & $0.0221,0.0217,0.0228$ & $1.6553,2.0772,0.3959$ & $0.0309,0.0223,0.0227$ \\
\hline
\end{tabular}

\section{CONCLUSION}

A two-component mixture model of the EIWD is considered in this article. In addition to the joint posterior distribution, the marginal posterior distributions for $\theta_{1}, \theta_{2}$ and $w$ are also derived assuming the uniform, Jeffreys, gamma, and inverse Levy priors. Moreover, to derive the Bayes estimates and their posterior risks, five different loss functions, namely, SELF, QLF, PLF, WLF, and WBLF have been considered. A real-life dataset is used for the selection of an appropriate loss function and of a prior. The results showed that the WBLF is a better loss function while the uniform prior is suitable for estimating $\theta_{1}, \theta_{2}$ and $w$, as the posterior risks for the Bayes estimators of $\theta_{1}, \theta_{2}$ and $w$ under WBLF were observed minimum assuming this prior. Similarly, the gamma prior is more informative than the inverse Levy prior. However, it is also observed that the informative priors have the minimum posterior risk as compared to the assumed loss functions. In future, Bayesian analysis for more than two-component mixture of the EIWD can be considered.

\section{REFERENCES}

AL-Hussaini E.K. \& Hussein M. (2012). Estimation under a finite mixture of exponentiated exponential components model and balanced square error loss. Open Journal of Statistics 2: $28-38$.

DOI: https://doi.org/10.4236/ojs.2012.21004

Ali S. (2014). Mixture of the inverse Rayleigh distribution: properties and estimation in Bayesian framework. Applied Mathematical Modelling 39(2): 515 - 530. DOI: https://doi.org/10.1016/j.apm.2014.05.039

Ali S., Aslam M. \& Kazmi S.M.A. (2012). On the Bayesian analysis of the mixture of Laplace distribution using the complete and the censored sample under different loss functions. Model Assisted Statistics and Applications 7(3): $209-227$.

Ali S., Aslam M. \& Kazmi S.M.A. (2014). Heterogeneous data analysis using mixture of Laplace model with conjugate priors. International Journal of Systems Science 45(12): $2619-2636$

DOI: https://doi.org/10.1080/00207721.2013.775381

Ahmad A., Ahmad S.P. \& Ahmad A. (2015). Bayesian estimation of inverted exponentiated Weibull distribution under asymmetric loss functions. Journal of Statistics: Applications and Probability 4: 183 - 192.

Aljuaid A. (2013). Estimating the parameters of an exponentiated inverted Weibull distribution under type II cencoring. Applied Mathematical Sciences 7: 1721 - 1736. DOI: https://doi.org/10.12988/ams.2013.13158

Cordeiro G.M., Ortega E.M.M. \& Cunha D.C.C. (2013). The exponentiated generalized class of distributions. Journal of Data Science 11: 1 - 27.

Datta D. (2013). Comparison of Weibull distribution and exponentiated Weibull distribution based estimation of mean and variance of wind data. International Journal of Energy, Information and Communications 4: 1 - 12 .

Elbatal I. \& Muhammed H.Z.(2014). Exponentiated generalized inverse Weibull distribution. Applied Mathematical Sciences 8: 3997 - 4012. DOI: https://doi.org/10.12988/ams.2014.44267

Flaih A., Elsalloukh H., Mendi E. \& Milanova M. (2012). The exponentiated inverse Weibull distribution. Applied Mathematics and Information Sciences 6: $167-171$.

Gupta R.D. \& Kundu D. (2001). Exponentiated exponential family: an alternative to Gamma and Weibull distributions. Biometrical Journal 43: 117 - 130.

Hassan A.S., Marwa A.A., Zaher H. \& Elsherpieny E.A. (2014). Comparison of estimators for exponentiated inverse Weibull distribution based on grouped data. Journal of Engineering Research and Applications 4: 77 - 90.

Lehmann E.L. (1953). The power of rank tests. The Annals of Mathematical Statistics 24: 23 - 43. 
DOI: https://doi.org/10.1214/aoms/1177729080

Lemonte A.J. \& Cordeiro J.M. (2011). The exponentiated generalized inverse Gaussian distribution. Statistics and Probability Letters 81: 506 - 517.

DOI: https://doi.org/10.1016/j.spl.2010.12.016

Li L.A. \& Sedransk N. (1988). Mixtures of distributions: a topological approach. The Annals of Statistics 16(4): $1623-1634$.

DOI: https://doi.org/10.1214/aos/1176351057

Murthy D.N.P., Xie M. \& Jiang R. (2004). Weibull Models. John Wiley and Sons, Inc., New York, USA.

Nadarajah S. \& Kotz S. (2006). The exponentiated type distributions. Acta Applicandae Mathematicae 92: 97-111.
DOI: https://doi.org/10.1007/s10440-006-9055-0

Rinne H. (2008). The Weibull Distribution: A Handbook. CRC Press, Florida, USA.

DOI: https://doi.org/10.1201/9781420087444

Sultan K.S., Ismail M.A. \& Al-Moisheer A.S. (2007). Mixture of two inverse Weibull distributions: properties and estimation. Computational Statistics and Data Analysis 51(1): 5377 - 5387.

DOI: https://doi.org/10.1016/j.csda.2006.09.016

Tahir M., Aslam M. \& Hussain Z. (2016). On the Bayesian analysis of 3-component mixture of exponential distribution under different loss functions. Hacettepe Journal of Mathematics and Statistics 45(2): $609-628$. 